\title{
1 GLP is critical for oogenesis exhibiting a G9A-independent role in
}

\section{transcriptional repression}

4 Hannah Demond ${ }^{1,2}$, Courtney W Hanna ${ }^{1,3,4}$, Juan Castillo-Fernandez ${ }^{1}$, Fátima Santos ${ }^{1,3}$, Evangelia K

$5 \quad$ Papachristou ${ }^{5}$, Anne Segonds-Pichon ${ }^{6}$, Kamal Kishore ${ }^{5}$, Clive S D'Santos ${ }^{5}$, Gavin Kelsey ${ }^{1,3,7 *}$

$6 \quad{ }^{1}$ Epigenetics Programme, Babraham Institute, Cambridge CB22 3AT, United Kingdom

7 2Laboratory of Integrative Biology, Scientific \& Technological Bioresource Nucleus Center for

8 Excellence in Translational Medicine (BIOREN-CEMT), Universidad de la Frontera, Temuco, 4810296,

$9 \quad$ Chile

$10{ }^{3}$ Centre for Trophoblast Research, University of Cambridge, Cambridge CB2 3EG, United Kingdom

$11{ }^{4}$ Department of Physiology, Development and Neuroscience, University of Cambridge, Cambridge CB2

12 3EG, United Kingdom

$13{ }^{5}$ Cancer Research UK Cambridge Institute, Li Ka Shing Centre, University of Cambridge, Robinson Way,

14 Cambridge CB2 ORE, United Kingdom

$15{ }^{6}$ Bioinformatics Group, Babraham Institute, Cambridge CB22 3AT, United Kingdom

$16{ }^{7}$ Wellcome-MRC Institute of Metabolic Science-Metabolic Research Laboratories, Cambridge CB2

17 OQQ, United Kingdom

*Corresponding author: Gavin Kelsey (gavin.kelsey@babraham.ac.uk) 


\section{Abstract}

GLP (EHMT1) functions as an H3K9me1 and H3K9me2 methyltransferase through its reportedly obligatory dimerization with G9A (EHMT2). Here, we investigated the role of GLP in oocyte and embryo development in comparison to G9A using oocyte-specific conditional knockout mouse models (G9a cKO, Glp cKO, G9a-Glp cDKO). Loss of GLP in oogenesis severely impairs oocyte maturation, fertilization and embryo development, resulting in lethality before embryonic day E12.5. In contrast, loss of G9A has a milder effect with a proportion of embryos producing viable offspring. The Glp cKO also showed loss of G9A protein and, hence, was phenotypically very similar to the G9a-Glp cDKO. H3K9me2 was equally depleted in all cKO genotypes, whereas H3K9me1 was decreased only in Glp cKO and G9a-Glp cDKO oocytes. Furthermore, the transcriptome, DNA methylome and proteome were markedly more affected in G9a-Glp cDKO than G9a cKO oocytes, demonstrating that in the absence of GLP there are widespread epigenetic and gene expression changes in the oocyte independent of $\mathrm{H} 3 \mathrm{~K} 9 \mathrm{me2}$. Gene dysregulation with coupled changes in DNA methylation suggest localised loss of chromatin repression, resulting in upregulated protein expression. Together, our findings demonstrate that GLP can function independently of G9A in the oocyte and is required for oocyte developmental competence.

\section{Keywords}




\section{Introduction}

41

42

The mammalian germline is the context for widespread epigenetic reprogramming, in which the somatic epigenetic signature is erased and replaced by a germline signature that is distinct in sperm and oocyte. Prior to the onset of gametogenesis, primordial germ cells undergo complete erasure of epigenetic marks, such as DNA methylation (Guibert et al. 2012; Seisenberger et al. 2012) and histone3 lysine-9 dimethylation (H3K9me2) (Seki et al. 2005). In the oocyte, reprogramming occurs postnatally during oocyte growth, resulting in a unique DNA methylation and histone modification landscape (Hanna et al. 2018a). As the oocyte does not divide during these processes, the oocyte can serve as a tractable system to study epigenetic regulation. Importantly, resetting of epigenetic marks is essential to support successful oogenesis, oocyte-to-embryo transition, as well as embryonic development (e.g. Kaneda et al. 2004; Andreu-Vieyra et al. 2010; Eymery et al. 2016; Kim et al. 2016; Xu et al. 2019).

G9A (EHMT2) and GLP (EHMT1) are best known as histone methyltransferases, although they modify non-histone targets as well. They preferentially function as heterodimers in vivo and comprise the main H3K9 mono- and di-methyltransferases in euchromatin (Tachibana et al. 2001; Peters et al. 2003; Rice et al. 2003; Tachibana et al. 2005; Tachibana et al. 2008). Both proteins contain a SET-domain, required for their catalytic activity as methyltransferases and heterodimer formation, as well as an ankyrin repeat domain that enables binding to H3K9me1 and H3K9me2 (Tachibana et al. 2001; Collins et al. 2008). They have been shown to be interdependent, as H3K9me1 and H3K9me2 levels are equally depleted in G9a KO and Glp KO embryonic stem cells (ESCs) (Tachibana et al. 2005). Moreover, G9a and Glp KO mouse models show very similar phenotypes with embryos displaying loss of H3K9me2, growth retardation and embryonic lethality between embryonic day (E)8.5 and E12.5 (Tachibana et al. 2002; Tachibana et al. 2005). As such, studies often do not distinguish between G9A and GLP, or solely consider G9A, resulting in limited knowledge of GLP function. However, recent 
findings indicate that GLP can act independently of G9A, revealing a role for GLP in targeting H3K27me2 to the paternal pronucleus in the zygote (Meng et al. 2020).

In the oocyte, loss of G9A impairs maturation and meiosis, with consequences for preimplantation lacking oocyte-derived G9A develop to term and result in healthy pups. The role of GLP in the oocyte and its impact on embryo development remain unclear. To investigate the importance of maternal GLP in the oocyte and embryo and compare its function to G9A, we used cKO mice for G9a and Glp, as well as a G9a-GIp cDKO. We compared the progression of oogenesis and embryogenesis in each cKO, and evaluated changes in H3K9 methylation, gene expression, DNA methylation and protein abundance in CKO oocytes. Our findings demonstrate that GLP has a unique and essential function in the oocyte.

\section{Results}

\section{GLP and G9a function during oocyte maturation and meiosis}

To analyse the function of GLP in oocytes and compare it to that of G9A, we generated three cKO models. Mice carrying floxed alleles for G9a (G9a cKO) (Sampath et al. 2007), Glp (Glp cKO) (Schaefer et al. 2009) or both G9a and Glp (G9a-Glp cDKO) were crossed with a Zp3-Cre driver, which is expressed exclusively in growing oocytes after postnatal day 5 (Lan et al. 2004). During oocyte maturation in the ovary, the germinal vesicle (GV) oocyte undergoes a change in chromatin conformation from a non-surrounded nucleolus (NSN) to a surrounded nucleolus (SN) stage that coincides with global transcriptional silencing. In control animals, immunofluorescence (IF) analysis showed that G9A and GLP are detected throughout the nucleus of NSN oocytes, but were no longer detectable by the mature SN stage (Supplemental Fig. S1A,B). In G9a cKO oocytes, G9A protein was lost in NSN oocytes, while GLP remained detectable (Fig. 1A). In contrast, Glp cKO oocytes were 
depleted for both G9A and GLP, similar to G9a-GIp cDKO oocytes, indicating that GLP is required for G9A stability but not vice versa, as was previously shown in mouse ESCs (Fig. 1A) (Tachibana et al. 2005).

Loss of G9A has been shown to affect oocyte maturation and meiosis (Au Yeung et al. 2019). To assess whether loss of GLP has additional effects on developmental capacity of the oocyte, we first analysed the NSN-to-SN maturation rate by staining fully-grown GV oocytes with DAPI and staging them according to chromatin conformation. In G/p cKO and G9a-Glp cDKO oocytes from 12-week old females the proportion of SN oocytes was significantly lower than in G9a cKO and control oocytes (Fig. 1B), indicating that loss of GLP has a stronger effect on oocyte maturation than loss of G9A alone.

To determine whether GLP is required for meiosis, we analysed spindle conformation and chromosome alignment of ovulated metaphase II (MII) oocytes after hormonal stimulation. In all three cKO models, an increase in abnormal chromatin configuration and spindle alignment was observed compared to controls, although variation between mice was high and differences did not reach statistical significance (Fig. 1C). Many meiotic abnormalities were observed, ranging from chromosomes that were located together but not aligned ("aggregates"), chromosome alignments where one or several chromosomes were misaligned, to chromosomes scattered throughout the nucleus (Supplemental Fig. S1C,D). Spindle abnormalities included collapsed, mono- or multipolar spindles (Fig. 1C and Supplemental Fig. S1C). Taken together, the results show that loss of G9A has mild effects on oocyte maturation compared to the significant impairment caused by loss of GLP, while effects on meiosis were similar.

\section{Loss of maternal GLP but not G9A results in prenatal developmental arrest}

To assess whether loss of GLP also compromises competence of oocytes, we examined developmental progression of embryogenesis. E3.5 embryos were collected from cKO females naturally mated with 
bioRxiv preprint doi: https://doi org/10.1101/2021.12.20.473460; this version posted December 21, 2021. The copyright holder for this preprint (which was not certified by peer review) is the author/funder, who has granted bioRxiv a license to display the preprint in perpetuity. It is made available under aCC-BY 4.0 International license.

wildtype (WT) C57BI/6Babr males and scored according to developmental stage. The majority of embryos from control females had reached the blastocyst stage (Fig. 1D). In comparison, fewer embryos derived from cKO oocytes progressed to blastocysts, with the proportion of maternal G9aGIp cDKO blastocysts significantly reduced, and the proportion of maternal G9a-Glp cDKO embryos arrested at the 1-cell stage significantly increased.

To determine the stage of embryo arrest, embryos were collected from superovulated, naturally mated cKO females at E1.5 and cultured in vitro for 3 days until day E4.5. Already at E1.5 a difference was observed, all three cKO models having a higher proportion of unfertilized oocytes and 1-cell embryos than controls. The G9a-Glp cDKO again showed the strongest phenotype, having significantly fewer 2-cell embryos than controls (Supplemental Fig. S2A). Fertilized embryos (1- and 2-cell) were selected for in vitro culture: none of the embryos from Glp cKO or G9a-Glp cDKO oocytes developed to blastocysts, arresting at earlier stages (1 to 4-cell stage) by E3.5 (Supplemental Fig. S2B,C). In contrast, $42.9 \%$ maternal G9a cKO embryos developed at least to morulae by E3.5 (Supplemental Fig. S2B,C). However, this was significantly less than controls where $91.1 \%$ of embryos reached morula or blastocyst stages by E3.5. These findings reveal that embryos derived from G9a cKO oocytes show reduced survival through preimplantation development, whereas preimplantation developmental competence of Glp cKO and G9a-Glp cDKO oocytes was severely impaired.

A small percentage of embryos from Glp cKO oocytes did progress in vivo to blastocysts, therefore we examined the implantation and development of embryos after natural mating. In line with findings above, there were significantly fewer implanted embryos at E6.5 from Glp cKO and G9a-Glp cDKO oocytes than G9a cKO and controls (Fig. 1E). At E8.5, 3 maternal Glp cKO and 6 G9a-Glp cDKO embryos were recovered: all were highly abnormal, with no clear tissue types or only extraembryonic tissue (Supplemental Fig. S2D,E). In contrast, although some abnormalities (predominantly developmental delay, but also abnormal morphology) were observed among E8.5 embryos from G9a cKO oocytes, most appeared normal (Supplemental Fig. S2D,E). By E12.5, 55\% (11/25) embryos from G9a cKO 
bioRxiv preprint doi: https://doi org/10.1101/2021.1220.473460; this version posted December 21, 2021. The copyright holder for this preprint (which was not certified by peer review) is the author/funder, who has granted bioRxiv a license to display the preprint in perpetuity. It is made available under aCC-BY 4.0 International license.

oocytes were grossly abnormal or exhibited developmental delay (Supplemental Fig. S2F,G). In G9aGlp cDKO females, with one exception, all embryos had died and only resorption sites were observed (Supplemental Fig. S2F).

Finally, we analysed the number of live pups born after mating cKO females with WT males: no pups were born to Glp cKO or G9a-Glp cDKO females, but G9a cKO females did give birth to a mean of 3.46 healthy pups per litter, although litter size was significantly reduced compared to controls (Fig. 1F). These results confirm previous findings in showing that although loss of G9A in the oocyte affects developmental capacity of pre- and post-implantation embryos, some embryos develop normally resulting in birth of healthy pups (Au Yeung et al. 2019). Our results show loss of GLP in the oocyte severely impairs embryonic development and although a small proportion of embryos reach the blastocyst stage and implant they die in utero between E8.5 and E12.5.

\section{Differential effects of loss of GLP or G9A on H3K9 methylation}

GLP and G9A are H3K9 methyltransferases required for establishment of H3K9me1 and H3K9me2 in euchromatin (Tachibana et al. 2001; Tachibana et al. 2002; Tachibana et al. 2005). Therefore, we examined H3K9 methylation in NSN-stage GV oocytes from cKO females (12 weeks) by IF. Consistent with previous reports (Peters et al. 2003; Rice et al. 2003), H3K9me1 and H3K9me2 were localized mainly in euchromatic chromatin of NSN oocytes, whereas H3K9me3 was enriched in heterochromatic foci (Fig. 2A). Fluorescence intensity of H3K9me1 was significantly reduced in Glp cKO and G9a-Glp cDKO oocytes compared to controls, but not in G9a cKO oocytes; in contrast, H3K9me2 decreased significantly in all three cKOs (Fig. 2A,B). A small but significant loss of H3K9me3 was observed in G9aGlp cDKO oocytes (Fig. 2A,B). Rather than a direct effect, this may result from loss of H3K9me1, as the H3K9me3 methyltransferase SUV39H requires H3K9me1 as a substrate (Pinheiro et al. 2012). 
bioRxiv preprint doi: https://doi.org/10.1101/2021.12.20.473460; this version posted December 21, 2021. The copyright holder for this preprint (which was not certified by peer review) is the author/funder, who has granted bioRxiv a license to display the preprint in perpetuity. It is made available under aCC-BY 4.0 International license.

With H3K9me1 and H3K9me2 affected by loss of G9A and/or GLP, we sought to evaluate the genomic localisation of these marks by ultra low-input native ChIP-seq (ULI-nChIP-seq) in WT GV oocytes from 15 and 25-day old females. We were able to obtain quality ChIP-seq libraries for H3K9me2 (Fig. 2C), but not for H3K9me1. By quantifying 10kb running windows, we observed a reproducible H3K9me2 enrichment between replicates from $\mathrm{d} 15$ and $\mathrm{d} 25$ WT oocytes, with $\mathrm{d} 25$ showing greater enrichment than d15 GV oocytes (Fig. 2C, Supplemental Fig. S3A). H3K9me2-enriched domains in d25 GV oocytes were defined by merging consecutive $10 \mathrm{~kb}$ windows with a $\log _{2} \mathrm{RPKM}>2.5(34,192$ or $12.5 \%$ of total 10kb windows; 12,514 domains; Fig. 2C, Supplemental Fig. S3B; Supplemental Table S1). To link H3K9me2 enrichment to transcription levels, we used published data to define CpG-island (CGI) and non-CGI promoters of low (FPKM $<0.1)$, medium (FPKM 0.1-1.0) and high (FPKM $>1.0)$ expressed genes (Veselovska et al. 2015), and then compared the overlap of these promoters with H3K9me2-enriched or random domains (Supplemental Fig. S3C). There was no difference in transcription level between genes localised to H3K9me2-enriched domains or random domains, thus H3K9me2 does not appear to act as a transcriptional repressor in oocytes.

We then assayed H3K9me2 in replicates of G9a cKO, G9a-Glp cDKO, and littermate control d25 GV oocytes. Notably, H3K9me2 libraries from control oocytes were $4.94 \%$ of total chromatin, whereas H3K9me2 libraries from G9a cKO and G9a-Glp cDKO oocytes were only $0.58 \%$ and $0.33 \%$, respectively (Fig. 2D), confirming that H3K9me2 is almost completely absent from cKO oocytes. Principal component analysis (PCA) of all biological replicates showed that G9a cKO and G9a-Glp cDKO H3K9me2 replicates clustered apart from control, d15 WT and d25 WT H3K9me2 replicates (Fig. 2E). When comparing enrichment across H3K9me2-enriched domains (+/-5kb), G9a cKO and G9a-Glp cDKO showed significant loss of H3K9me2 compared to controls, with enrichment comparable to that in the IgG control (Fig. 2F). Furthermore, this effect was specific to H3K9me2-enriched domains, as there was no observable difference in enrichment between cKOs and controls across a set of random domains (Fig. 2F). There was a similar loss of H3K9me2 in G9a cKO and G9a-Glp cDKO oocytes, supporting the IF results that G9A and GLP are both required for $\mathrm{H} 3 \mathrm{~K} 9 \mathrm{me} 2$ in oogenesis. 
Beside their role as histone methyltransferases, G9A and GLP are known to methylate non-histone proteins and, by doing so, can potentially modulate their function, localisation or stability. To assess whether loss of GLP affects protein abundance in the oocyte, we performed low-input (200 oocytes) quantitative whole-proteome mass spectrometry isobaric labelling analysis of control, G9a cKO and G9a-Glp cDKO GV oocytes. In total, 21,358 peptides were detected at FDR $<1 \%$ and 3,182 quantified proteins (Supplemental Table S2). Gene ontology (GO) analysis showed enrichment for processes involved in cellular localization and organization, as well as protein folding, metabolic processes and fertilization (Fig. 3A). Furthermore, among the top 50 most abundant proteins, we detected oocytespecific proteins such as members of the subcortical maternal complex (PADI6, NLRP5, NLRP14, TLE6, KHDC3, NLRP4F), the zona pellucida (ZP1, ZP2, ZP3), as well as proteins known to be highly abundant in the oocyte (DNMT1, UHRF1).

Comparing G9a-Glp cDKO with control oocytes, we identified 187 proteins with a significant change in abundance ( $P<0.05$ and $\log _{2} \mathrm{FC}>0.3$; Fig. 3B-D, Supplemental Table S2). In contrast, there were only 38 differentially abundant proteins in G9a cKO oocytes, of which 21 overlapped with those in G9a-Glp cDKO oocytes. The majority of changing proteins increased in abundance in both G9a cKO (12 down, 26 up) and G9a-Glp cDKO oocytes (12 down, 175 up; Fig. 3B,C). Although few proteins were identified to have significant changes in abundance in G9a cKO oocytes, many proteins changing in G9a-G/p cDKO oocytes displayed the same directional trend in G9a cKO oocytes (Fig. 3B). these were significantly regulated in CKO oocytes; this is not entirely unexpected as protein methylation does not necessarily influence abundance, but rather may affect activity. GO analysis did not detect any significant enrichment terms amongst the differentially abundant proteins. Even so, 
bioRxiv preprint doi: https://doi org/10.1101/2021.12.20.473460; this version posted December 21, 2021. The copyright holder for this preprint (which was not certified by peer review) is the author/funder, who has granted bioRxiv a license to display the preprint in perpetuity. It is made available under aCC-BY 4.0 International license.

211

212

213

we identified several proteins for which change in abundance may be related to the observed oocyte phenotypes. Among these, two were meiotic factors according to the MGI Gene Ontology Browser (CDC25B and SIRT1), while literature research linked a further seven (STAT3, TGON1, SET, IMPDH2, DSTN, SKA3, ROCK1) to meiosis in the oocyte (Supplemental Table S2). Furthermore, proteins involved in oocyte maturation (ERMP1) and fertilization (ASTL) showed significantly increased abundance, as well as oocyte transcription factor HMGB3. Increased abundance of these proteins may underlie the meiotic spindle abnormalities, impaired oocyte maturation and poor fertilization rates observed in G9a-GIp cKO oocytes. In line with observations in oocyte and embryo development, as well as H3K9 methylation, the more severe effect in G9a-Glp cDKO oocytes suggests an additional role for GLP. The similar but lesser, non-significant changes in protein abundance observed in G9a cKO oocytes suggests that the function of GLP may be compensatory of G9A, rather than unique.

\section{Loss of GLP results in local and distinct DNA methylation changes}

G9A-mediated H3K9me2 has been linked to DNA methylation (Tachibana et al. 2008; Zeng et al. 2019), although genome-wide analysis only detected local DNA methylation changes in G9a cKO oocytes and G9a KO embryos (Auclair et al. 2016; Au Yeung et al. 2019). To examine whether loss of GLP affects DNA methylation establishment in the oocyte, we first analysed global DNA methylation (5mC and $5 \mathrm{hmC}$ ) by IF. In NSN oocytes, $5 \mathrm{mC}$ was observed throughout the nucleus in both euchromatic and heterochromatic regions (Fig. 4A); $5 \mathrm{hmC}$ was enriched in heterochromatic foci but also present in euchromatin (Fig. 4A). No changes in localization of $5 \mathrm{mC}$ or $5 \mathrm{hmC}$ were observed in cKO oocytes. However, when assessing $5 \mathrm{mC}$ fluorescence intensity, there were significant decreases in the Glp cKO and G9a-GIp cDKO compared to control oocytes, but not for G9a cKO oocytes (Fig. 4B). No significant changes in $5 \mathrm{hmC}$ levels were observed in cKO oocytes, although $5 \mathrm{hmC}$ levels also appear reduced in cKO oocytes. These data suggest that de novo DNA methylation may be impaired upon loss of GLP but not G9A in oocytes. 
bioRxiv preprint doi: https://doi org/10.1101/2021.1220.473460; this version posted December 21, 2021. The copyright holder for this preprint (which was not certified by peer review) is the author/funder, who has granted bioRxiv a license to display the preprint in perpetuity. It is made available under aCC-BY 4.0 International license.

We then explored changes in DNA methylation in greater resolution by whole-genome bisulphite sequencing (BS-seq) of control, G9a cKO and G9a-GIp cDKO GV oocytes. In contrast to decreased $5 \mathrm{mC}$ seen by IF, BS-seq did not show significant global changes in DNA methylation (Supplemental Fig. S4A). An explanation for this apparent discrepancy is that the genomic regions assessed by the two methods differ: IF signal reports both euchromatic and heterochromatic fractions of the genome, while BS-seq data is enriched in euchromatic and non-repetitive regions that can be uniquely mapped and analysed. These differences highlight the value of using both methods to understand genomewide patterns of DNA methylation and may explain previous contradictory findings (Au Yeung et al. 2019; Zeng et al. 2019).

Although global DNA methylation levels as detected by BS-seq did not differ, local changes in DNA methylation were analysed by binning the genome into consecutive windows of $100 \mathrm{CpG}$ sites (100CpG windows). Differential methylation analysis identified 9,187 DMRs in G9a-Glp cDKO oocytes (4.88\% of all analysed $100-$ CpG windows), of which 4,184 (45.5\%) were hypermethylated (mean methylation difference: $35.1 \%)$ and 5,003 (54.5\%) hypomethylated (mean methylation difference 25.6\%) (Fig. 4C; Supplemental Table S3). Methylation was more affected in G9a-Glp cDKO than in G9a cKO oocytes, with $>7$ times more DMRs identified: there were 1,252 DMRs $(0.67 \%$ of all analysed 100 CpG windows) in the G9a cKO, of which 432 (34.5\%) were hypermethylated (mean methylation difference: $39.4 \%)$ and 820 (65.5\%) hypomethylated (mean methylation difference: 33.8\%) (Supplemental Fig. S4B). Importantly, the majority of G9a cKO DMRs overlapped G9a-Glp cDKO DMRs (Supplemental Fig. 4C). This indicates that while both G9A and GLP are required for normal DNA methylation establishment in the oocyte, GLP can partially compensate for loss of G9A, but it does not exclude the possibility that GLP has an additional G9A-independent role in DNA methylation.

To assess this possibility in more detail, we analysed the regions of the genome affected by DNA methylation changes. While hypermethylated DMRs show similar overlap with genic and intergenic regions, hypomethylated DMRs are enriched in genic regions (Supplemental Fig. S4D). This is 
bioRxiv preprint doi: https://doi org/10.1101/2021.1220.473460; this version posted December 21,2021 . The copyright holder for this preprint (which was not certified by peer review) is the author/funder, who has granted bioRxiv a license to display the preprint in perpetuity. It is made available under aCC-BY 4.0 International license.

261

262

263

264

265

266

267

268

269

270

271

272

273

274

275

276

277

278

expected, as hypomethylated DMRs are found in regions that are methylated in control oocytes and it is well established that DNA methylation localises to transcribed genes in oocytes (Veselovska et al. 2015). To assess whether DNA methylation changes correlate with underlying histone modifications, we tested the overlap of DMRs with H3K9me2, H3K27me3 and H3K4me3 (Fig. 4D). The majority of DMRs did not overlap regions with H3K9me2 and there was no clear distinction between hyper- and hypomethylated DMRs, indicating that most DNA methylation changes in cKO oocytes are unlikely to be a direct consequence of loss of H3K9me2. Interestingly, hypermethylated DMRs were strongly enriched for H3K27me3, compared to hypomethylated DMRs. Importantly, this enrichment was not seen for H3K4me3, although both are enriched in regions of the genome lacking DNA methylation. Because H3K27me3 and DNA methylation are mutually exclusive, this result may indicate a localised redistribution of the two marks in cKOs.

Since G9A and GLP can interact with UHRF1 (Kim et al. 2009; Ferry et al. 2017), we also investigated the overlap of DMRs with regions that are hypomethylated in Uhrf1 cKO oocytes (Maenohara et al. 2017). Uhrf1 cKO oocytes show widespread hypomethylation, and we observed that both G9a cKO and G9a-GIp cDKO hypomethylated DMRs strongly overlap with Uhrf1 hypomethylated regions compared to random and random methylated probes (Fig. 4E). These data support that loss of G9A and GLP may disturb a subset of UHFR1-mediated methylation.

Between 21 and $38 \%$ of $100-C p G$ windows identified as DMRs cluster and form larger regions that, in both genotypes, can span genes (Fig. 4E, Supplemental Fig. S4E-G and Supplemental Table S3). In total, there were 707 hypo- and 869 hyper-methylated domains in G9a-Glp cDKO oocytes, with fewer in the G9a cKO (140 hypo- and 66 hyper-methylated domains). To see whether the additional changes in the G9a-Glp cDKO are unique or whether similar (non-significant) trends are present in the $G 9 a$ cKO, we performed unsupervised cluster analysis of DNA methylation of the CDKO domains (Fig. 4F). For both hypo- and hypermethylated domains, we identified domains common to both cKOs (523 hypo, 590 hyper) and domains unique to the G9a-Glp cDKO (229 hypo, 279 hyper; Fig. 4F,G; 
Supplemental Fig. S4E-G). This indicates that the majority of effects seen in the G9a-Glp cDKO are also present in the G9a cKO, although to a lower, often non-significant magnitude. Furthermore, there appears to be a potentially unique role of GLP in oocyte DNA methylation, although this is likely to be minor compared to its function compensatory to loss of G9A.

\section{Transcriptome changes direct DNA methylation and protein changes in G9a-Glp cDKO oocytes}

We evaluated gene expression changes by RNA-seq in G9a-GIp cDKO, G9a cKO and matched control GV oocytes. Differential gene expression was determined by DESeq2 analysis followed by filtering for genes with $\log _{2} \mathrm{FC}>1.5$. The vast majority of differentially expressed genes (DEGs) were upregulated in the G9a-GIp cDKO. Again, G9a-GIp cDKO oocytes were more severely affected than G9a cKO oocytes, with 330 DEGs (301 up, 29 down; Fig. 5A, Supplemental Table S4), in contrast to 79 DEGs in G9a cKO oocytes (64 up, 15 down; Supplemental Fig. S5A). Of the G9a cKO DEGs, 51 overlapped with G9a-Glp cDKO DEGs. Clustering analysis identified three clusters: 1) downregulated in both G9a cKO and G9a-Glp cDKO; 2) upregulated in both genotypes; and 3) uniquely upregulated in G9a-Glp cDKO (Fig. 5B). GO analysis did not show significant category enrichments, but amongst the deregulated transcripts we identified several transcription factors with a known function in the oocyte (Prmt7, Etv5), zygotic genome activation (Zscan4d) and embryo development (KIf4, Hoxd1, Lmx1a; Supplemental Table S4). Furthermore, several genes important for oocyte maturation, meiosis and fertilization were deregulated (Atrx, Fgfr2, Prkcq, Ptgs2, Plac1, Mt1), which may underlie some of the phenotypic effects we see.

Both our proteome and transcriptome analysis showed a preferential upregulation of protein and transcript abundance in G9a-Glp cDKO oocytes, respectively. To assess whether changes in transcript expression may be causative for changes in protein abundance, we used gene set enrichment analysis (Fig. 5C). Indeed, proteins with increased abundance were enriched for transcriptionally upregulated genes in G9a-Glp cDKO oocytes, whereas proteins with decreased abundance were enriched for 
bioRxiv preprint doi: https://doi org/10.1101/2021.12.20.473460; this version posted December 21, 2021. The copyright holder for this preprint (which was not certified by peer review) is the author/funder, who has granted bioRxiv a license to display the preprint in perpetuity. It is made available under aCC-BY 4.0 International license.

downregulated genes, although this may not account for all the variation in protein abundance. This indicates that loss of GLP results in deregulated gene expression, which in turn affects abundance of the corresponding proteins. Hence, although the majority of genes appear unaffected by loss of GLP, the transcriptional changes that do occur are likely to be of biological significance.

Using the DEG clusters, we assessed the overlap of DEGs with the distribution of histone modifications

(Supplemental Fig. S5B). There was no significant enrichment for any of the clusters with H3K9me2, further supporting that $\mathrm{H} 3 \mathrm{~K} 9 \mathrm{me} 2$ does not correlate with transcriptional repression in the oocyte (Supplemental Fig. S3C). Interestingly, similar to hypermethylated DMRs, upregulated DEGs (clusters 2 and 3) were enriched in H3K27me3. As transcription is linked to the deposition of gene body DNA methylation in the mouse oocyte, we analysed the correlation between expression and DNA methylation changes. Consistently, expression changes of DEGs positively correlated with gene-body DNA methylation changes in G9a-Glp cDKO oocytes (Fig. 5D). In contrast, when evaluating genes with differential methylation, the correlation was much weaker (Supplemental Fig. S5C). These trends were similar in G9a cKO oocytes (Supplemental Fig. S5D,E). This shows that the transcriptional changes observed in G9a-GIp cDKO oocytes impact the associated genic DNA methylation; however, this association does not explain the majority of DNA methylation changes seen in G9a-Glp cDKO oocytes, thus other mechanisms likely underlie the majority of changes observed.

\section{Discussion}

Our present study shows that GLP can partially compensate for loss of G9A and additionally has a G9Aindependent role in oogenesis. We find that GLP is required for oocyte maturation, meiosis and developmental competence. Few embryos derived from Glp cKO oocytes implant and those that do die mid-gestation. In contrast, maternal G9a cKO embryos are less affected and some develop normally into healthy pups. This difference in severity is also reflected at a molecular level in gene expression, protein abundance and DNA methylation. Importantly, the differences between the 
bioRxiv preprint doi: https://doi org/10.1101/2021.12.20.473460; this version posted December 21, 2021. The copyright holder for this preprint (which was not certified by peer review) is the author/funder, who has granted bioRxiv a license to display the preprint in perpetuity. It is made available under aCC-BY 4.0 International license.

genotypes are independent of GLP's function as an H3K9me2 methyltransferase, as H3K9me2 was equally ablated in all. Therefore, we propose that the phenotypes arise from multiple mechanistic changes in histone modifications, transcriptional regulation and DNA methylation, summarized in Fig. 6.

To consider the molecular mechanisms underlying the observed phenotypes, it is important to appreciate that G9A and GLP are proteins with multiple functions. Although best known as histone methyltransferases, they can methylate and alter the function of non-histone proteins (Shinkai and Tachibana 2011; Scheer and Zaph 2017). Nevertheless, the strong correlation we observed between changes in transcript and protein abundance in $G 9 a$ and $G 9 a / G / p$ cKO oocytes supports that the changes in protein abundance are likely attributable to altered genomic regulation rather than G9A/GLP directly modulating protein stability. Loss of G9A and GLP resulted in transcriptional upregulation, supporting previous reports that G9A/GLP act as repressors (Tachibana et al. 2002; Tachibana et al. 2005). It is unlikely that this can be attributed mostly to loss of H3K9me2, because only a fraction of genes was upregulated despite almost complete ablation of H3K9me2, and because of the significant transcriptional differences between the G9a cKO and G9a-G/p cDKO despite similar deficits in H3K9me2. Alternatively, the effects may be mediated indirectly by G9A/GLP modulating activity of transcriptional repressors or through other chromatin changes. We also observed upregulation of some developmental transcription factors, suggesting that some gene expression changes reflect illegitimate expression of such factors in oocytes. The more extensive transcriptional deregulation in the G9a-Glp cDKO is associated with impaired developmental capacity of the oocyte, resulting in decreased oocyte maturation, meiotic spindle abnormalities, impaired fertilization and abnormal embryo development.

G9A and GLP have been linked to DNA methylation in multiple studies. In the embryo, loss of G9A leads to hypomethylation of a subset of CGI promoters (Auclair et al. 2016). In the oocyte, we and others do not see widespread hypomethylation, but instead local sites of hypo- and hypermethylation 
bioRxiv preprint doi: https://doi.org/10.1101/2021.12.20.473460; this version posted December 21, 2021. The copyright holder for this preprint (which was not certified by peer review) is the author/funder, who has granted bioRxiv a license to display the preprint in perpetuity. It is made available under aCC-BY 4.0 International license.

361 (Au Yeung et al. 2019). A variety of mechanisms could cause these changes. In the oocyte, de novo DNA methylation requires transcription (Kobayashi et al. 2012; Veselovska et al. 2015). The transcriptional changes in G9a cKO and G9a-Glp cDKO oocytes correlate with DNA methylation changes, indicating that upregulated gene expression is responsible for some of the hypermethylation observed. However, the significant localisation of G9a cKO and G9a-GIp cDKO hypermethylated regions with H3K27me3 suggests that the loss of G9A/GLP may impair the repressive chromatin landscape in the oocyte, either through $\mathrm{H} 3 \mathrm{~K} 9 \mathrm{me} 2$ or modulation of a transcriptional repressor. Conversely, a large subset of hypomethylated DMRs overlapped with regions that are hypomethylated in Uhrf1 cKO oocytes (Maenohara et al. 2017). UHRF1 function has been associated with G9A and GLP in several ways, either by direct interaction (Kim et al. 2009) or indirect recruitment through H3K9me2/3 or LIG1 (Rothbart et al. 2012; Rothbart et al. 2013; Ferry et al. 2017). However, it is not clear how these mechanisms apply in the oocyte because of the absence of DNA replication. Although UHRF1 appears to be required for some genomic DNA methylation, loss of DNMT1 has only minor effects, largely through a role in ensuring symmetric methylation of de novo methylated CpGs (Shirane et al. 2013; Maenohara et al. 2017). There are likely other mechanisms besides transcriptional regulation and UHRF1 interactions at play in the G9a cKO and G9a-Glp cDKO oocytes. These include the possibility of a direct interaction of G9A and GLP with DNMT proteins. For example, G9A and GLP can dimethylate DNMT3A at lysine 44 . Methylated DNMT3A can be bound by MPP8, which in turn binds automethylated GLP, resulting in a DNMT3A-MPP8-GLP silencing complex (Chang et al. 2011). In contrast to previous reports (Tachibana et al. 2005), we saw that while H3K9me2 depends on both G9A and GLP, the persistence of GLP in the G9a cKO was sufficient to establish H3K9me1. Notably, loss of G9A and GLP did not completely eliminate H3K9me1, indicating that other methyltransferases, such as PRDM3 and PRDM16, may be active in the oocyte. It remains unclear whether the decrease in H3K9me1 may be (partially) causative for the transcriptional and DNA methylation changes: so far, H3K9me1 has not been associated with either but rather is proposed to be predominantly a substrate for H3K9 di- and trimethylation (Pinheiro et al. 2012). 
It is difficult to distinguish between a compensatory effect and a unique role for GLP in the oocyte. oocytes, some genes/proteins/regions are progressively affected while others only affected in G9ain most DEGs was reflected in an upregulation of protein abundance in G9a-Glp cDKO oocytes, indicating that although only a relatively small proportion of genes is derepressed upon loss of G9A and GLP, the transcriptional changes are likely to have a functional impact in the oocyte. Indeed, we saw changes in gene expression and protein abundance of several genes related to oocyte maturation, meiosis and fertilization. Taken together, our results highlight GLP as a multi-functional protein involved in different regulatory processes in the oocyte. They show that it has a unique role that is critical for the developmental capacity of the oocyte and independent of G9A and its role as H3K9me2 methyltransferase.

\section{Materials and methods}

\section{Sample collections}

All experimental procedures were performed under licences issued by the Home Office (UK) in accordance with the Animals (Scientific Procedures) Act 1986 and were approved by the Animal

Welfare and Ethical Review Body at the Babraham Institute.

Oocytes and embryos were collected in M2 medium (Sigma-Aldrich, M7167) unless stated otherwise. 
and RNA-seq were collected from ovaries of 22-26 day-old mice using a collagenase/trypsin digest. For ChIP-seq, $300 \mathrm{GV}$ oocytes were collected in nuclear lysis buffer and pooled from two to four mice for each replicate. For BS-seq and RNA-seq, replicates were stored in RLT+ buffer (Qiagen). Each replicate comprised all oocytes collected from one mouse (75-200 GV oocytes). For whole-proteome analysis oocytes were collected from ovaries of 22-26 day-old mice. To avoid contamination with proteins/peptides from the medium, oocytes were collected by manual dissection of ovaries in protein-free L15 medium (Thermo Fisher Scientific, 31415029). 200 GV oocytes were collected from two mice in parallel, washed 3 times in PBS with 1x cOmplete Protease Inhibitor Cocktail (Merck, 11697498001).

Preimplantation embryos were collected after natural mating of control and cKO females with implantation sites in uteri at E6.5 after timed mating. To score postimplantation development, embryos were collected after natural mating at E8.5 and E12.5. For embryo culture, female mice were superovulated and embryos dissected in M2 medium at E1.5 after natural mating with WT males. developmental progress recorded each day.

429 IF was performed after antibody staining as previously described (Santos et al. 2003). Primary 430 antibodies are listed in Supplemental Table S5. Samples were analysed on a Zeiss LSM780 confocal 431 microscope (63x oil-immersion objective). Single optical sections were captured and semi432 quantification of fluorescence intensity was performed using Volocity 6.3 (Improvision). 
bioRxiv preprint doi: https://doi.org/10.1101/2021.1220.473460; this version posted December 21, 2021. The copyright holder for this preprint (which was not certified by peer review) is the author/funder, who has granted bioRxiv a license to display the preprint in perpetuity. It is made available under aCC-BY 4.0 International license.

Oocytes were lysed in $20 \mu \mathrm{l}$ dissolution buffer containing $100 \mathrm{mM}$ triethylammonium bicarbonate (Sigma, T4708) and 0.1\% Sodium Dodecyl Sulfate (SDS), followed by water bath sonication and boiling at $90^{\circ} \mathrm{C}$ for $5 \mathrm{~min}$. Proteins were reduced with tris-2-carboxyethyl phosphine (TCEP, Sigma) for $1 \mathrm{~h}$ at $60^{\circ} \mathrm{C}$ at a final concentration of $5 \mathrm{mM}$, followed by cysteine blocking for $10 \mathrm{~min}$ at room temperature using methyl methanethiosulfonate (MMTS, Sigma) at final concentration of $10 \mathrm{mM}$. Samples were digested overnight at $37^{\circ} \mathrm{C}$ with trypsin (Pierce \#90058) and the next day peptides were labelled with TMT11plex reagents (0.4mg per sample) according to manufacturer's instructions (Thermo Scientific). To quench the reaction, $3 \mu$ of $5 \%$ hydroxylamine (Thermo Scientific) was added for $15 \mathrm{~min}$ and samples combined and dried with centrifugal vacuum concentrator. The TMT mix was fractionated with Reversed-Phase spin columns at high pH (Pierce \#84868). Nine fractions were collected using different elution solutions in the range of $5-50 \%$ ACN and were analysed on a Dionex UltiMate 3000 UHPLC system coupled with the nano-ESI Fusion-Lumos (Thermo Scientific) mass spectrometer. Samples were loaded on the Acclaim PepMap 100, $100 \mu \mathrm{m} \times 2 \mathrm{~cm} \mathrm{C18}, 5 \mu \mathrm{m}, 100 \dot{A}$ trapping column with the ulPickUp injection method at loading flow rate $5 \mu \mathrm{l} / \mathrm{min}$ for $10 \mathrm{~min}$. For peptide separation, the EASY-Spray analytical column $75 \mu \mathrm{m} \times 25 \mathrm{~cm}, \mathrm{C} 18,2 \mu \mathrm{m}, 100 \dot{A}$ column was used for multi-step gradient elution. Full scans were performed in the Orbitrap in the range of $380-1500 \mathrm{~m} / \mathrm{z}$ at $120 \mathrm{~K}$ resolution and peptides isolated in the quadrupole with isolation window 1.2Th, HCD collision energy $38 \%$ and resolution 50K. Raw data were processed with the SequestHT search engine in Proteome Discoverer 2.1 software and searched against a Uniprot database containing mouse reviewed entries. The parameters for the SequestHT node were: Precursor Mass Tolerance 20ppm, Fragment Mass Tolerance $0.02 \mathrm{Da}$, Dynamic Modifications were Oxidation of $\mathrm{M}(+15.995 \mathrm{Da})$, Deamidation of $\mathrm{N}, \mathrm{Q}$ (+0.984Da) and Static Modifications were TMT6plex at any N-Terminus, K (+229.163Da) and Methylthio at C (+45.988Da). The consensus workflow included TMT signal-to-noise $(\mathrm{S} / \mathrm{N})$ calculation and the level of confidence for peptide identifications was estimated using the Percolator node with decoy database search. Strict FDR was set at $q$-value $<0.01$. For downstream data analysis, the $R$ package qPLEXanalyzer was used (Papachristou et al. 2018). The mass spectrometry proteomics data 
have been deposited to the ProteomeXchange Consortium via the PRIDE partner repository (Perez-

Riverol et al. 2019) with the dataset identifier PXD030265.

\section{Preparation of sequencing libraries}

ChIP-seq libraries were prepared using ULI-nChIP as previously described (Hanna et al. 2018b).

Antibodies were added at 250ng/reaction for both anti-H3K9me2 (mouse monoclonal, Abcam, ab1220) and anti-IgG (rabbit polyclonal, Diagenode, EB-070-010). Library preparation was completed with a MicroPlex Library Preparation kit v2 (Diagenode) with Sanger 8-base indices for multiplexing. Relative enrichment over input was quantified using the library concentrations determined by Bioanalyzer High Sensitivity DNA Analysis (Agilent). Low input bisulphite (BS)-seq libraries were prepared by post-bisulphite adapter tagging as previously described (Hanna et al. 2018b). RNA-seq libraries were prepared as described (Hanna et al. 2019).

\section{Sequencing and data processing}

472 Libraries were sequenced on Illumina MiSeq, HiSeq2500 and NextSeq500 systems. ChIP-seq libraries

473 were sequenced to an average of 57 million paired-end reads of $75 \mathrm{bp}$ read-length (Supplemental

474 Table S5). BS-seq libraries were sequenced to an average of 16 million paired-end reads for G9a cKO

475 and 26 million reads for G9a-Glp cDKO oocytes of 100-125 bp read-length. RNA-seq libraries were sequenced to an average single read number of 1.9 million for G9a cKO and G9a-Glp cDKO oocytes of

47750 bp read-length. Raw fastq files were processed with Trim Galore, then mapped to the mouse guided by known splice sites, BS-seq data with Bismark v0.19.0.

Sequencing data analysis 
bioRxiv preprint doi: https://doi org/10.1101/2021.12.20.473460; this version posted December 21, 2021. The copyright holder for this preprint (which was not certified by peer review) is the author/funder, who has granted bioRxiv a license to display the preprint in perpetuity. It is made available under aCC-BY 4.0 International license.

484

485

486

487

488

489

490

491

492

493

494

495

496

497

498

499

500

501

502

503

504

505

506

507

508

were filtered to exclude mapping artefacts, defined as RPKM>6 in at least one replicate set of $10 \%$ input libraries $(N=408)$. H3K9me2 enrichment was defined as $\log _{2} R P K M>2.5$ in d25 GV oocytes $(N=34,192)$. A set of random windows was sampled from all 10kb windows $(N=35,000)$. H3K9me2enriched and random windows were then merged with adjacent windows within $10 \mathrm{~kb}$, resulting in 12,154 H3K9me2-enriched domains and 26,077 random domains. Genic and intergenic regions were defined as overlapping or not overlapping oocyte transcripts, respectively, and promoters were defined as +/-500bp around transcription start sites of oocyte transcripts (Veselovska et al. 2015). CGls were defined as previously described (Illingworth et al. 2008). Oocyte transcription levels were categorized into not expressed (FPKM $<0.1)$, low expressed (FPKM 0.1-1) and high expressed (FPKM $>1$ ) using published data (Veselovska et al. 2015).

BS-seq data were analysed using a tile-based approach of $100 \mathrm{CpGs}$ for each consecutive genome window, ensuring equal $C p G$ content in all windows analysed. Methylation values were quantified using the bisulphite-sequencing pipeline quantification, which calculates per-base methylation percentages and averages these within each window. Filters were applied to ensure a minimum coverage of $\geq 10$ observed cytosines per window. Only windows with this minimal coverage in all samples were taken into account, allowing assessment of $86.2 \%$ of $100 \mathrm{CpG}$ windows $(N=188,433)$. Differentially methylated regions (DMRs) were defined by statistical comparison of DNA methylation levels for each $100 \mathrm{CpG}$ window between control and G9a cKO or control and G9a-GIp cDKO oocytes using the EdgeR function in SeqMonk. To assess overlap of DMRs with genomic features, CGI and oocyte gene annotations were used from (Veselovska et al. 2015). DMRs were merged to form differentially methylated domains (DMDs). Hierarchical clustering analysis of DMDs was performed in $\mathrm{R}$ using Euclidean distance and Ward's agglomeration method as implemented by the package pheatmap.

For analysis of RNA-seq data, expression of oocyte genes (Veselovska et al. 2015) was quantified using log transformed read count quantitation per million reads. Differential gene expression was analysed 
using DESeq2 followed by filtering of genes with $\log _{2} \mathrm{FC}>1.5$. Clustering analysis of DEGs was performed in R using Euclidean distance and Ward's agglomeration method. Relative enrichment of differentially abundant proteins amongst DEGs was displayed using the barcode method from the Limma R package. The ranked list of expression changes only considered genes corresponding to proteins identified in the proteome. Spearman's rank correlation coefficient between DNA methylation difference and expression $\log _{2} \mathrm{FC}$ was used to interrogate the relationship between DNA methylation and transcriptional changes. ChIP-seq and BS-seq data under accessions GSE93941, DRA005849 were used to develop our studies.

\section{Statistical analysis}

Statistical analysis was conducted in Graphpad Prism, R and VassarStats. SN proportion was compared using two-way ANOVA (Stage x Genotype) with Šídák's multiple comparisons test. Proportion of embryo stage at E3.5, number of implantation sites at E6.5 and mean fluorescence intensity of confocal IF images were analysed using one-way ANOVA with Tukey's posthoc test. Litter sizes were compared using a nested one-way ANOVA with mouse as nested factor. A $\chi$-square test was used to analyse frequency of overlap of genic/intergenic regions with DMRs, histone modifications with DMRs, Uhrf1 hypomethylated regions with DMRs and DEGs with histone modifications. $P$-value was adjusted using Bonferroni correction to control for multiple comparisons.

\section{$528 \quad$ Figure legends}

Figure 1. Developmental potential of G9a cKO, Glp cKO and G9a-Glp cDKO oocytes.

A) Representative images showing IF for G9A and GLP in NSN oocytes. Scale bar: $20 \mu \mathrm{m}$. 
$P=0.7667$; Ctrl vs Glp cKO: $P=0.0075 ;$ Ctrl vs G9a-Glp cDKO: $P<0.0001 ;$ G9a cKO vs G9a-Glp cDKO $P=0.0058$.

C) Bar charts showing percentage of chromosome misalignments and spindle abnormalities in MII oocytes. Dots represent datapoints from single mice. Examples of normal and abnormal spindles are shown in IF images below. G9a cKO: $P=0.7037$; Glp cKO: $P=0.0678$; G9a-Glp cDKO: $P=0.0670$.

D) Stacked bar chart showing developmental stage (\%) of embryos from cKO females mated with WT males and collected on E3.5. Number of mice: Control=5, G9a cKO=5, Glp cKO=6, G9a-Glp cDKO=6. Blastocysts: Ctrl vs G9a cKO P=0.9939; Ctrl vs Glp cKO $P=0.0761$, Ctrl vs G9a-Glp cDKO $P=0.0073$; One-cell stage: Ctrl vs G9a-Glp cDKO $P=0.0406$.

E) Bar chart showing number of implantation sites scored at E6.5. Dots represent single mice. Ctrl vs Glp cKO P=0.0093; Ctrl vs G9a-Glp cDKO $P=0.0020$.

F) Boxplots showing average litter size of females with cKO oocytes mated with WT males. Number of mice: Control=9, $G 9 a$ cKO=5, $G / p$ cKO=3, G9a-Glp cDKO=3; Number of litters: Control=60, $G 9 a$

Figure 2. Analysis of H3K9 methylation in G9a cKO, Glp cKO and G9a-Glp cDKO oocytes.

A) Representative IF images of NSN oocytes for H3K9me1, H3K9me2 and H3K9me3 in control, G9a cKO, Glp cKO and G9a-Glp cDKO oocytes.

B) Boxplots of quantitation of IF images. Dots represent individual oocytes. Analysis is based on 2-5 mice per genotype and 3-4 replicate experiments for each antibody.

C) Genome screenshot showing H3K9me2 enrichment as determined by ChIP-seq in WT oocytes from mice aged 15 and 25 days. Y-axis scaling represents RPKM. Annotation tracks highlight oocyte transcripts, CpG islands, oocyte DNA methylated and unmethylated domains, and H3K9me2enriched domains ( $\log _{2}$ RPKM $>2.5$ in d25 GV oocytes).

D) Boxplots showing relative enrichment over input of H3K9me2 ChIP-seq libraries. Dots represent individual samples. 
E) PCA plot of H3K9me2 (blue shading), input (green shading) and IgG (yellow shading) ChIP-seq libraries, comparing WT (d15 and d25), control, G9a cKO and G9a-Glp cDKO samples.

F) Probe trend plot showing loss of H3K9me2-enrichment in G9a cKO and G9a-Glp cDKO oocytes compared to WT and control oocytes in H3K9me2-enriched domains but not random domains.

563

Figure 3. Proteome analysis of G9a cKO and G9a-Glp cDKO oocytes.

A) GO analysis of the oocyte proteome.

B) Volcano plot showing differential abundance of proteins in G9a cKO oocytes compared to controls. Significantly changing proteins $\left(P<0.05\right.$ and $\left.\log _{2} \mathrm{FC}>0.3\right)$ are highlighted in dark blue (decreased abundance) or dark red (increased abundance); light blue and light red dots indicate proteins changing significantly in cDKO oocytes.

C) Volcano plot showing differential abundance of proteins in G9a-Glp cDKO oocytes compared to controls. Significantly changing proteins $\left(P<0.05\right.$ and $\left.\log _{2} \mathrm{FC}>0.3\right)$ are highlighted in dark blue (decreased abundance) or dark red (increased abundance). Light blue and light red dots indicate proteins changing significantly in G9a cKO oocytes. Proteins changing significantly and with oocyte function of interest are labelled.

D) Venn diagram of significant changing proteins in G9a cKO and G9a-GIp cDKO oocytes.

Figure 4. DNA methylation changes in G9a cKO and G9a-Glp cDKO oocytes.

A) Representative IF images of NSN oocytes showing $5 \mathrm{mC}$ and $5 \mathrm{hmC}$ in Control, G9a cKO, Glp cKO and G9a-Glp cDKO oocytes.

B) Boxplots of quantitation of IF images. Dots represent individual oocytes. Analysis is based on 2 mice per genotype and 2 replicate experiments for each antibody. Unique cDKO DMRs are highlighted in blue, DMRs shared with G9a cKO oocytes in red. 
D) Barchart showing percentage overlap of DMRs and random probes with H3K9me2, H3K37me3 and H3K4me3. H3K9me2: G9a cKO hyper adj. $P=0.0136$, G9a cKO hypo $P<0.0001, G 9 a-G / p$ CDKO hypo $P<0.0001$; H3K27me3: G9a cKO hyper $P<0.0001$, G9a-Glp cDKO hyper $P<0.0001$; H3K4me3: all genotypes $P<0.0001$.

E) Barchart showing percentage overlap of DMRs with 100-CpG windows that are hypomethylated in Uhrf1 cKO oocytes (>20\% methylation difference, (Maenohara et al. 2017)), random 100-CpG windows and 100-CpG windows that are highly methylated (>75\%) in WT and Uhrf1 cKO oocytes. G9a cKO hyper adj. P=0.0404, G9a-Glp cDKO hyper, G9a cKO hypo and G9a-Glp cDKO hyper $P<0.0001$.

F) Genome screenshot showing example of a region uniquely hypomethylated in G9a-Glp cDKO oocytes spanning the Arhgef1O/ transcript. Annotation tracks show position of H3K9me2-enriched domains and oocyte transcripts.

G) Heatmap showing clustering analysis of G9a-Glp cDKO hypo- and hypermethylated domains.

597

Figure 5. Transcription changes in G9a-GIp cDKO oocytes in relation to protein and DNA methylation changes.

A) Scatterplot showing expression levels in control vs. G9a-Glp cDKO oocytes. Unique cDKO DEGs are highlighted in blue, DEGs with $\log _{2} \mathrm{FC}>1.5$ in red and DEGs shared with G9a cKO oocytes in green.

B) Heatmap showing relative expression levels (RPKM) of up- and down-regulated G9a-Glp cDKO DEGs that overlap with G9a cKO DEGs.

C) Plot showing link between changes in transcript and protein abundance. The $\mathrm{X}$-axis shows $\log _{2} \mathrm{FC}$ $(N=2933)$. Blue and red boxes represent down- and up-regulated transcripts, respectively, with a FC $>1.5$ ( $\left.\log _{2} \mathrm{FC}>0.585\right)$. Vertical blue and red lines represent differentially abundant proteins $\left(P<0.05\right.$ and $\log _{2} \mathrm{FC}>0.3$; ten proteins not detected in the RNA-seq data are not represented). 
among downregulated transcripts and upregulated proteins enriched among upregulated transcripts (Spearman correlation $R=0.43 ; P=2.2 \times 10^{-9}$ ).

D) Plot showing correlation between methylation changes (\% methylation difference) and expression changes ( $\log _{2} \mathrm{FC}$ ) of DEGs in G9a-Glp cDKO oocytes. Relative expression levels (RPKM) of each gene are indicated by the colour scale. Spearman correlation is shown.

615

Figure 6. The distinct roles of GLP and G9A in the oocyte.

A) Summary of the developmental and molecular phenotypes of G9a cKO, Glp cKO and G9a-Glp cDKO oocytes.

B) Proposed activities of GLP homodimers and G9A/GLP heterodimers on H3K9 methylation.

C) Interaction of GLP and/or G9A/GLP with UHRF1 could explain DNA hypomethylation in CKO and cDKO oocytes.

D) Alternative chromatin changes that could contribute to gene up-regulation in G9a cKO and G9aGlp cDKO oocytes.

\section{Competing interest statement}

The authors declare no competing interests.

\section{Acknowledgements}

We would like to Kristina Tabbada and Nicole Forrester of the Sequencing, Simon Walker of the Imaging, Simon Andrews and Felix Krueger of the Bioinformatics facilities, and members of the Biological Support Unit at the Babraham Institute for excellent support. Work in G.K.'s lab was funded by the UK Biotechnology and Biological Sciences Research Council (BBS/E/B/000C0423) and Medical Research Council (MR/K011332/1, MR/S000437/1); H.D. was supported by a Fondecyt Postdoctorado 
Trophoblast Research. Part of the work in C.D'S.'s lab was funded by CRUK core grant (A29580) awarded to the Cambridge Institute.

Author contributions: C.H., H.D. and G.K. conceptualised the study. H.D., F.S., E.K.P. and C.H. collected the data. C.H., H.D., J.C-F., and K.K. performed data analysis. C.H., H.D. and J.C.-F. generated manuscript figures. H.D. drafted the manuscript, with input from C.H., J.C.-F., E.K.P., C.D'S. and G.K.

\section{References}

Andreu-Vieyra CV, Chen R, Agno JE, Glaser S, Anastassiadis K, Stewart AF, Matzuk MM. 2010. MLL2 is required in oocytes for bulk histone 3 lysine 4 trimethylation and transcriptional silencing. PLOS Biol 8.

Au Yeung WK, Brind'Amour J, Hatano Y, Yamagata K, Feil R, Lorincz MC, Tachibana M, Shinkai Y, Sasaki H. 2019. Histone H3K9 Methyltransferase G9a in Oocytes Is Essential for Preimplantation Development but Dispensable for CG Methylation Protection. Cell reports 27: 282-293 e284.

Auclair G, Borgel J, Sanz LA, Vallet J, Guibert S, Dumas M, Cavelier P, Girardot M, Forne T, Feil R et al. 2016. EHMT2 directs DNA methylation for efficient gene silencing in mouse embryos. Genome Res 26: 192-202.

Chang Y, Sun L, Kokura K, Horton JR, Fukuda M, Espejo A, Izumi V, Koomen JM, Bedford MT, Zhang X et al. 2011. MPP8 mediates the interactions between DNA methyltransferase Dnmt3a and H3K9 methyltransferase GLP/G9a. Nat Commun 2: 533.

Collins RE, Northrop JP, Horton JR, Lee DY, Zhang X, Stallcup MR, Cheng X. 2008. The ankyrin repeats of G9a and GLP histone methyltransferases are mono- and dimethyllysine binding modules. Nat Struct Mol Biol 15: 245-250.

Eymery A, Liu Z, Ozonov EA, Stadler MB, Peters AH. 2016. The methyltransferase Setdb1 is essential for meiosis and mitosis in mouse oocytes and early embryos. Development.

Ferry L, Fournier A, Tsusaka T, Adelmant G, Shimazu T, Matano S, Kirsh O, Amouroux R, Dohmae N, Suzuki T et al. 2017. Methylation of DNA Ligase 1 by G9a/GLP Recruits UHRF1 to Replicating DNA and Regulates DNA Methylation. Mol Cell 67: 550-565 e555.

Guibert S, Forne T, Weber M. 2012. Global profiling of DNA methylation erasure in mouse primordial germ cells. Genome Res 22: 633-641.

Hanna CW, Demond H, Kelsey G. 2018a. Epigenetic regulation in development: is the mouse a good model for the human? Hum Reprod Update 24: 556-576.

Hanna CW, Perez-Palacios R, Gahurova L, Schubert M, Krueger F, Biggins L, Andrews S, ColomeTatche M, Bourc'his D, Dean W et al. 2019. Endogenous retroviral insertions drive noncanonical imprinting in extra-embryonic tissues. Genome biology 20: 225.

Hanna CW, Taudt A, Huang J, Gahurova L, Kranz A, Andrews S, Dean W, Stewart AF, Colome-Tatche M, Kelsey G. 2018b. MLL2 conveys transcription-independent H3K4 trimethylation in oocytes. Nat Struct Mol Biol 25: 73-82.

Illingworth R, Kerr A, Desousa D, Jorgensen H, Ellis P, Stalker J, Jackson D, Clee C, Plumb R, Rogers J et al. 2008. A novel CpG island set identifies tissue-specific methylation at developmental gene loci. PLoS Biol 6: e22. 
Kaneda M, Okano M, Hata K, Sado T, Tsujimoto N, Li E, Sasaki H. 2004. Essential role for de novo DNA methyltransferase Dnmt3a in paternal and maternal imprinting. Nature 429: 900-903.

Kim J, Zhao H, Dan J, Kim S, Hardikar S, Hollowell D, Lin K, Lu Y, Takata Y, Shen J et al. 2016. Maternal Setdb1 Is Required for Meiotic Progression and Preimplantation Development in Mouse. PLoS Genet 12: e1005970.

Kim JK, Esteve PO, Jacobsen SE, Pradhan S. 2009. UHRF1 binds G9a and participates in p21 transcriptional regulation in mammalian cells. Nucleic Acids Res 37: 493-505.

Kobayashi H, Sakurai T, Imai M, Takahashi N, Fukuda A, Yayoi O, Sato S, Nakabayashi K, Hata K, Sotomaru $Y$ et al. 2012. Contribution of intragenic DNA methylation in mouse gametic DNA methylomes to establish oocyte-specific heritable marks. PLoS Genet 8: e1002440.

Lan ZJ, Xu X, Cooney AJ. 2004. Differential oocyte-specific expression of Cre recombinase activity in GDF-9-iCre, Zp3cre, and Msx2Cre transgenic mice. Biol Reprod 71: 1469-1474.

Maenohara S, Unoki M, Toh H, Ohishi H, Sharif J, Koseki H, Sasaki H. 2017. Role of UHRF1 in de novo DNA methylation in oocytes and maintenance methylation in preimplantation embryos. PLoS Genet 13: e1007042.

Meng TG, Zhou Q, Ma XS, Liu XY, Meng QR, Huang XJ, Liu HL, Lei WL, Zhao ZH, Ouyang YC et al. 2020. PRC2 and EHMT1 regulate H3K27me2 and H3K27me3 establishment across the zygote genome. Nat Commun 11: 6354.

Papachristou EK, Kishore K, Holding AN, Harvey K, Roumeliotis TI, Chilamakuri CSR, Omarjee S, Chia KM, Swarbrick A, Lim E et al. 2018. A quantitative mass spectrometry-based approach to monitor the dynamics of endogenous chromatin-associated protein complexes. Nat Commun 9: 2311.

Perez-Riverol Y, Csordas A, Bai J, Bernal-Llinares M, Hewapathirana S, Kundu DJ, Inuganti A, Griss J, Mayer G, Eisenacher $\mathrm{M}$ et al. 2019. The PRIDE database and related tools and resources in 2019: improving support for quantification data. Nucleic Acids Res 47: D442-D450.

Peters AH, Kubicek S, Mechtler K, O'Sullivan RJ, Derijck AA, Perez-Burgos L, Kohlmaier A, Opravil S, Tachibana M, Shinkai $Y$ et al. 2003. Partitioning and plasticity of repressive histone methylation states in mammalian chromatin. Mol Cell 12: 1577-1589.

Pinheiro I, Margueron R, Shukeir N, Eisold M, Fritzsch C, Richter FM, Mittler G, Genoud C, Goyama S, Kurokawa $\mathrm{M}$ et al. 2012. Prdm3 and Prdm16 are H3K9me1 methyltransferases required for mammalian heterochromatin integrity. Cell 150: 948-960.

Rice JC, Briggs SD, Ueberheide B, Barber CM, Shabanowitz J, Hunt DF, Shinkai Y, Allis CD. 2003. Histone methyltransferases direct different degrees of methylation to define distinct chromatin domains. Mol Cell 12: 1591-1598.

Rothbart SB, Dickson BM, Ong MS, Krajewski K, Houliston S, Kireev DB, Arrowsmith CH, Strahl BD. 2013. Multivalent histone engagement by the linked tandem Tudor and PHD domains of UHRF1 is required for the epigenetic inheritance of DNA methylation. Genes Dev 27: 12881298.

Rothbart SB, Krajewski K, Nady N, Tempel W, Xue S, Badeaux Al, Barsyte-Lovejoy D, Martinez JY, Bedford MT, Fuchs SM et al. 2012. Association of UHRF1 with methylated H3K9 directs the maintenance of DNA methylation. Nat Struct Mol Biol 19: 1155-1160.

Sampath SC, Marazzi I, Yap KL, Sampath SC, Krutchinsky AN, Mecklenbrauker I, Viale A, Rudensky E, Zhou MM, Chait BT et al. 2007. Methylation of a histone mimic within the histone methyltransferase G9a regulates protein complex assembly. Mol Cell 27: 596-608.

Santos F, Zakhartchenko V, Stojkovic M, Peters A, Jenuwein T, Wolf E, Reik W, Dean W. 2003. Epigenetic marking correlates with developmental potential in cloned bovine preimplantation embryos. Current biology : $C B$ 13: 1116-1121.

Schaefer A, Sampath SC, Intrator A, Min A, Gertler TS, Surmeier DJ, Tarakhovsky A, Greengard P. 2009. Control of cognition and adaptive behavior by the GLP/G9a epigenetic suppressor complex. Neuron 64: 678-691. 
Scheer S, Zaph C. 2017. The Lysine Methyltransferase G9a in Immune Cell Differentiation and Function. Front Immunol 8: 429.

Seisenberger S, Andrews S, Krueger F, Arand J, Walter J, Santos F, Popp C, Thienpont B, Dean W, Reik W. 2012. The dynamics of genome-wide DNA methylation reprogramming in mouse primordial germ cells. Mol Cell 48: 849-862.

Seki Y, Hayashi K, Itoh K, Mizugaki M, Saitou M, Matsui Y. 2005. Extensive and orderly reprogramming of genome-wide chromatin modifications associated with specification and early development of germ cells in mice. Dev Bio/ 278: 440-458.

Shinkai Y, Tachibana M. 2011. H3K9 methyltransferase G9a and the related molecule GLP. Genes Dev 25: 781-788.

Shirane K, Toh H, Kobayashi H, Miura F, Chiba H, Ito T, Kono T, Sasaki H. 2013. Mouse oocyte methylomes at base resolution reveal genome-wide accumulation of non-CpG methylation and role of DNA methyltransferases. PLoS Genet 9: e1003439.

Tachibana M, Matsumura Y, Fukuda M, Kimura H, Shinkai Y. 2008. G9a/GLP complexes independently mediate $\mathrm{H} 3 \mathrm{~K} 9$ and DNA methylation to silence transcription. EMBO $\mathbf{2 7}$ : 2681-2690.

Tachibana M, Sugimoto K, Fukushima T, Shinkai Y. 2001. Set domain-containing protein, G9a, is a novel lysine-preferring mammalian histone methyltransferase with hyperactivity and specific selectivity to lysines 9 and 27 of histone H3. J Biol Chem 276: 25309-25317.

Tachibana M, Sugimoto K, Nozaki M, Ueda J, Ohta T, Ohki M, Fukuda M, Takeda N, Niida H, Kato H et al. 2002. G9a histone methyltransferase plays a dominant role in euchromatic histone $\mathrm{H} 3$ lysine 9 methylation and is essential for early embryogenesis. Genes Dev 16: 1779-1791.

Tachibana M, Ueda J, Fukuda M, Takeda N, Ohta T, Iwanari H, Sakihama T, Kodama T, Hamakubo T, Shinkai Y. 2005. Histone methyltransferases G9a and GLP form heteromeric complexes and are both crucial for methylation of euchromatin at H3-K9. Genes Dev 19: 815-826.

Veselovska L, Smallwood SA, Saadeh H, Stewart KR, Krueger F, Maupetit-Mehouas S, Arnaud P, Tomizawa S, Andrews S, Kelsey G. 2015. Deep sequencing and de novo assembly of the mouse oocyte transcriptome define the contribution of transcription to the DNA methylation landscape. Genome biology 16: 209.

Xu Q, Xiang Y, Wang Q, Wang L, Brind'Amour J, Bogutz AB, Zhang Y, Zhang B, Yu G, Xia W et al. 2019. SETD2 regulates the maternal epigenome, genomic imprinting and embryonic development. Nat Genet 51: 844-856.

Zeng TB, Han L, Pierce N, Pfeifer GP, Szabo PE. 2019. EHMT2 and SETDB1 protect the maternal pronucleus from 5mC oxidation. Proc Natl Acad Sci U S A 116: 10834-10841. 
bioRxiv preprint doi: https://doi.org/10.1101/2021.12 20.473460; this version posted December 21, 2021. The copyright holder for this preprint (which was not certified by peer review) is the author/funder, who has granted bioRxiv a license to display the preprint in perpetuity. It is made available under aCC-BY 4.0 International license.

Figure 1

A

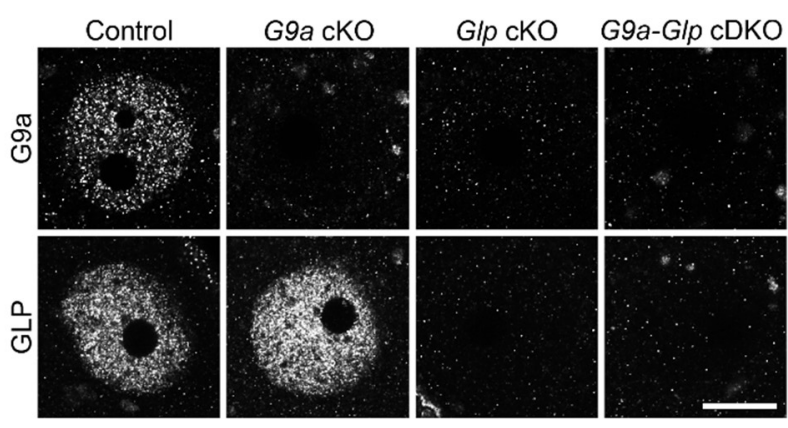

B

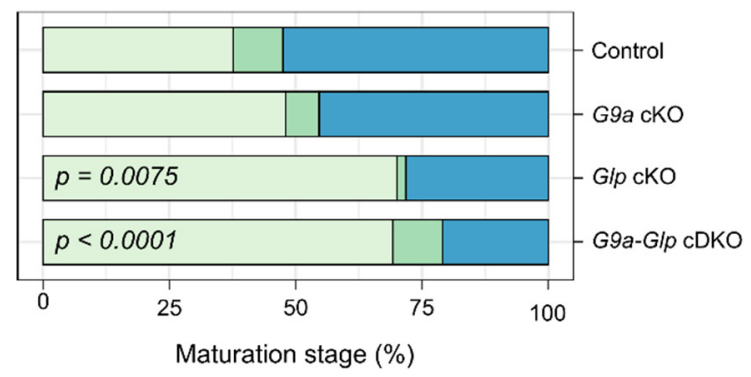

$\square$ NSN $\square$ Intermediate $\square$ SN

D

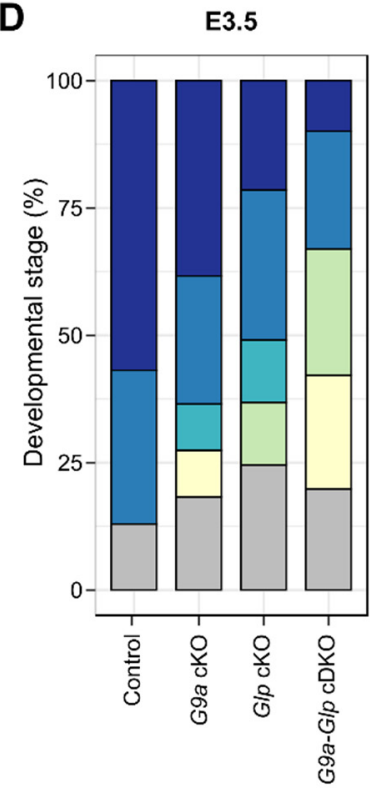

E
C
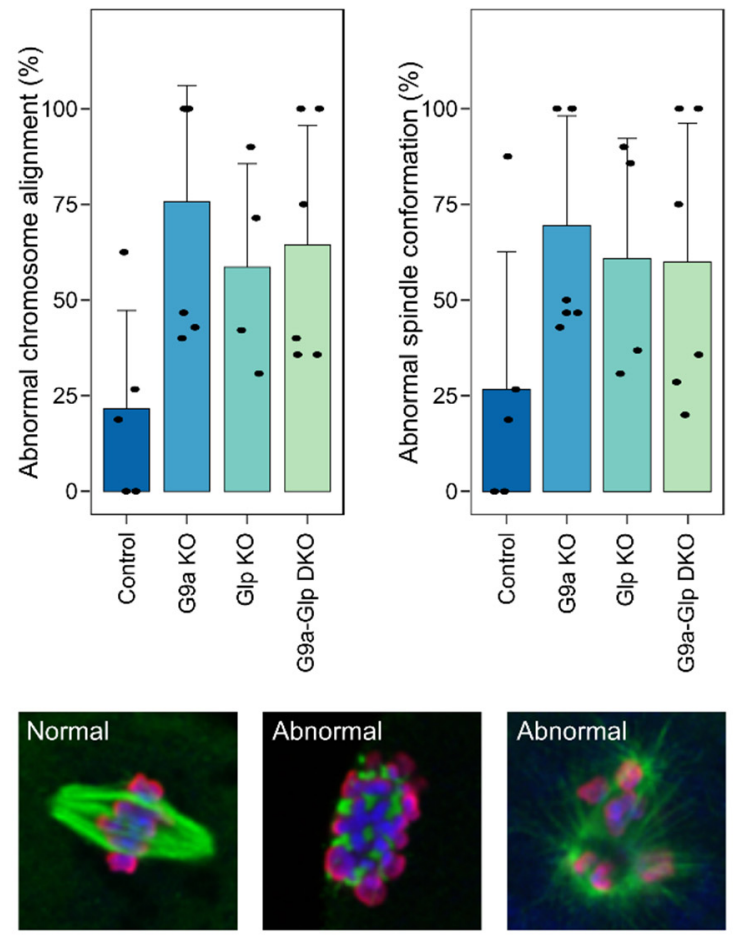

E6.5

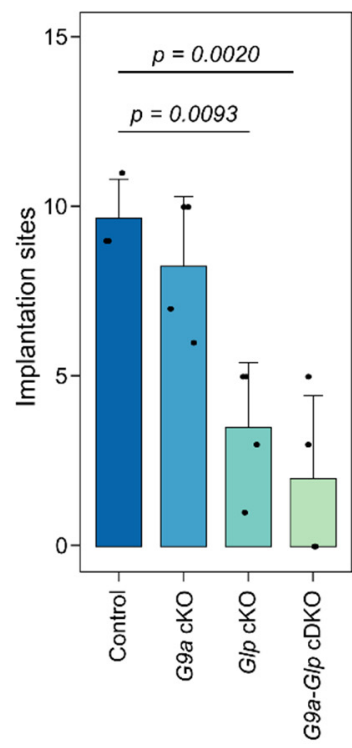

$\mathbf{F}$

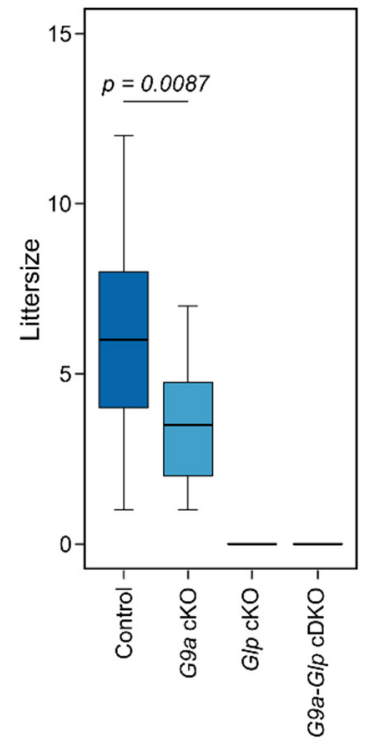


bioRxiv preprint doi: https://doi.org/10.1101/2021.12.20.473460; this version posted December 21, 2021. The copyright holder for this preprint (which was not certified by peer review) is the author/funder, who has granted bioRxiv a license to display the preprint in perpetuity. It is made available under aCC-BY 4.0 International license.

Figure 2

A

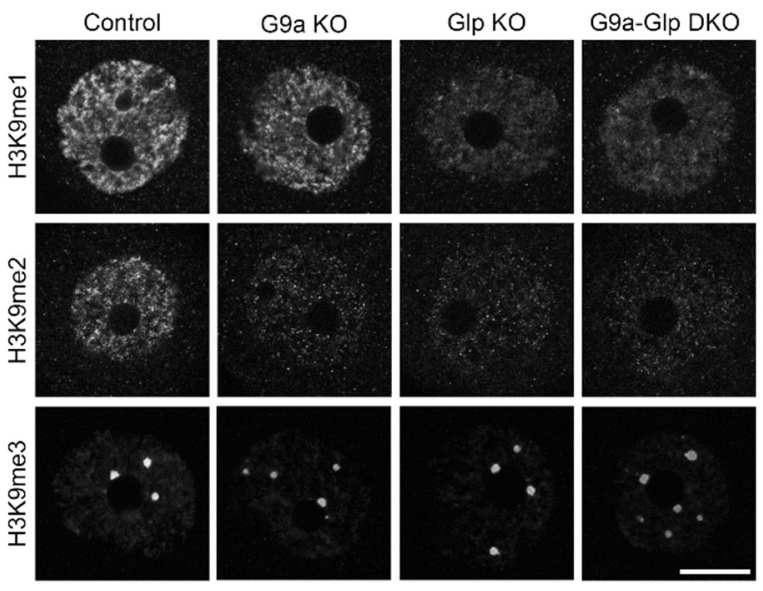

B

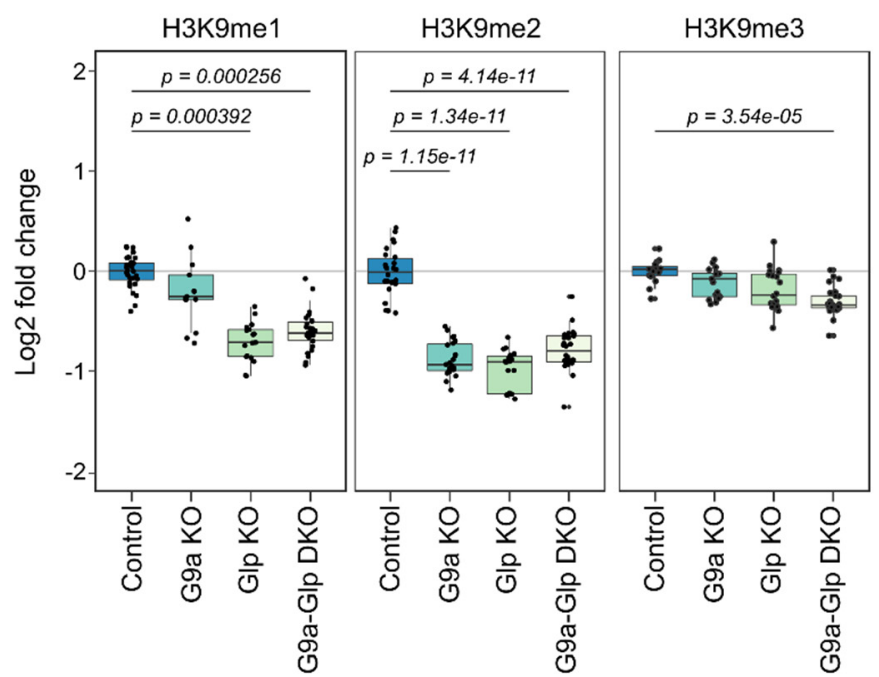

C

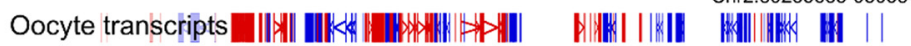

CpG islands $\quad||||||||||||||||||||||||||||||||||||||||||||||||||||$

GV meth./unmeth. dom. $\mathrm{III \|}$

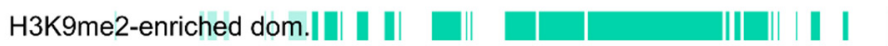

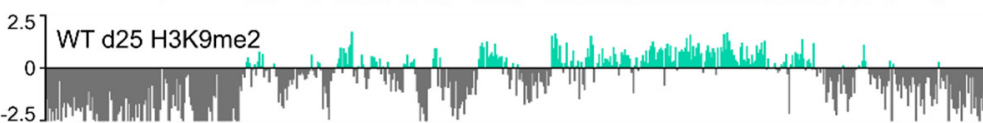

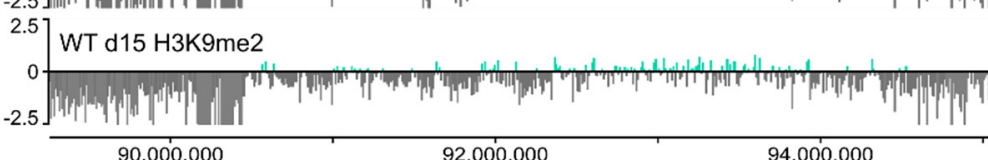

$92,000,000$

E

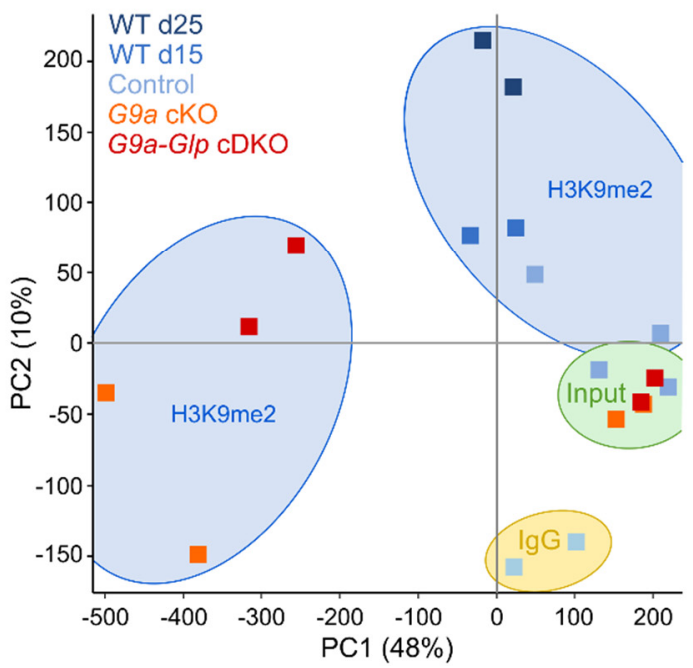

$94,000,000$

$\mathbf{F}$

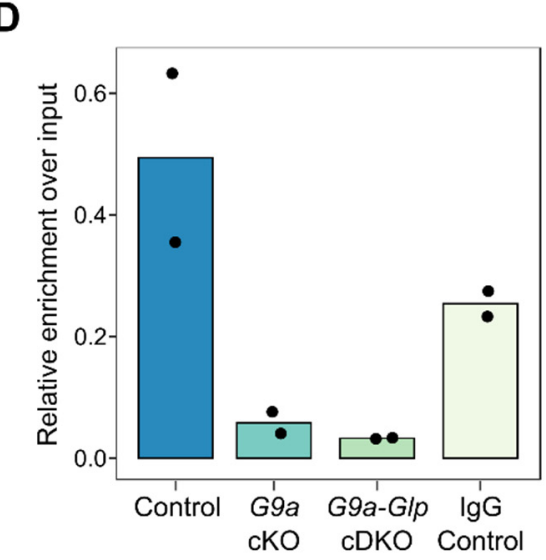

H3K9me2-enriched domains

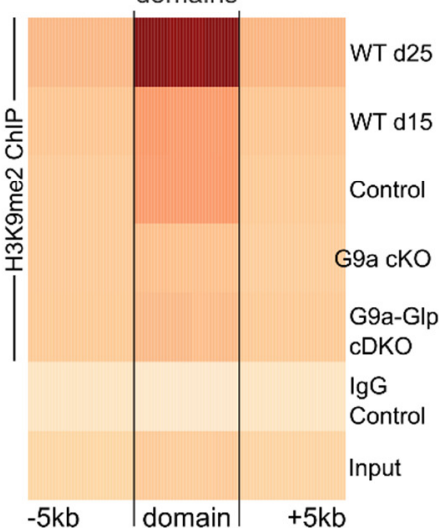

Random domains

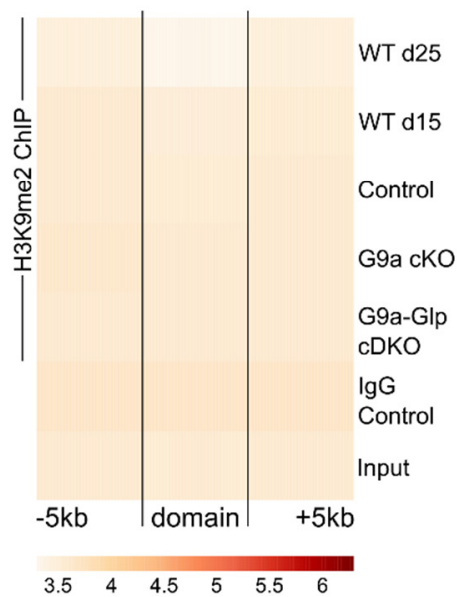


bioRxiv preprint doi: https://doi org/10.1101/2021.12 20.473460: this version posted December 21, 2021. The copyright holder for this preprint (which was not certified by peer review) is the author/funder, who has granted bioRxiv a license to display the preprint in perpetuity. It is made available under aCC-BY 4.0 International license.

\section{Figure 3}

A

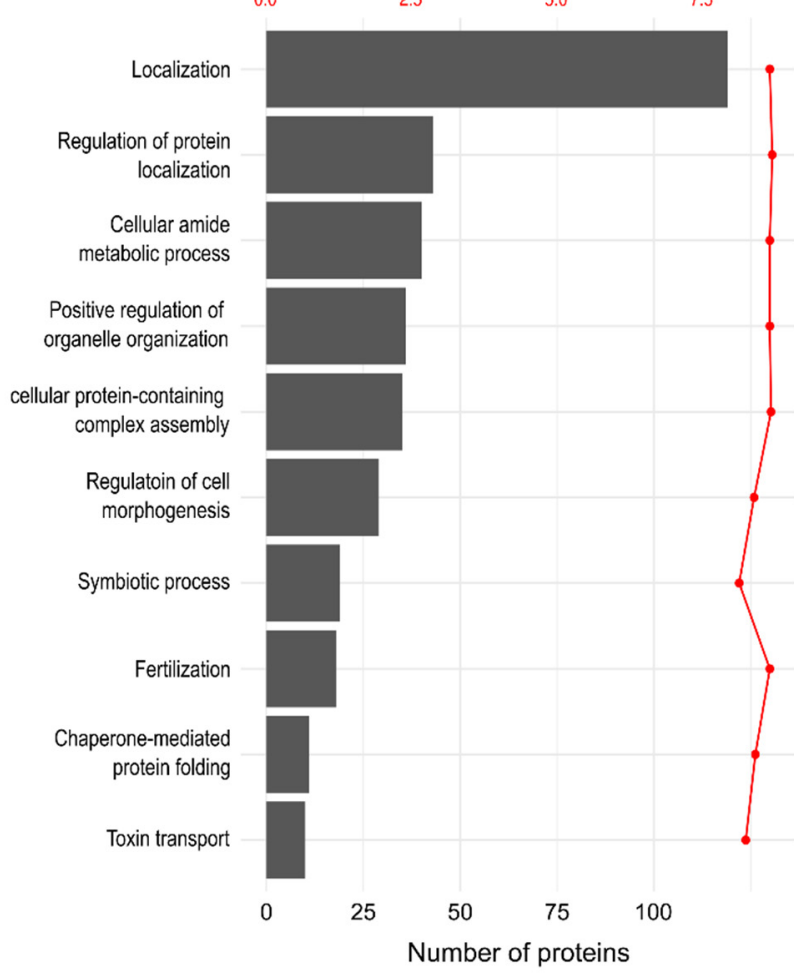

D

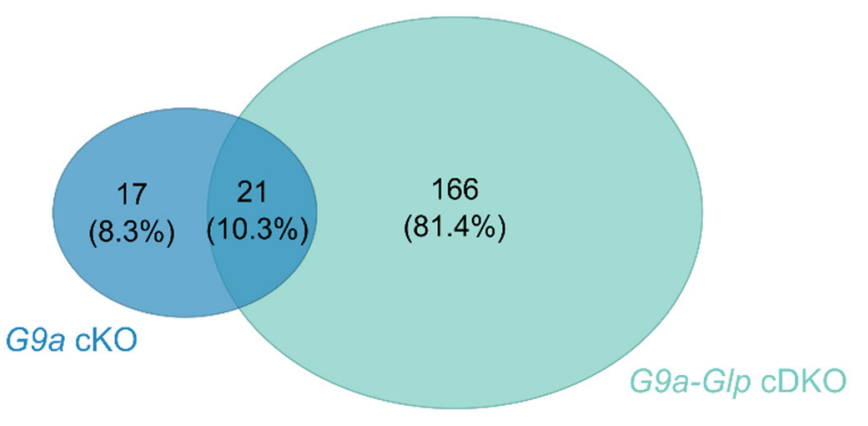

B G9acko

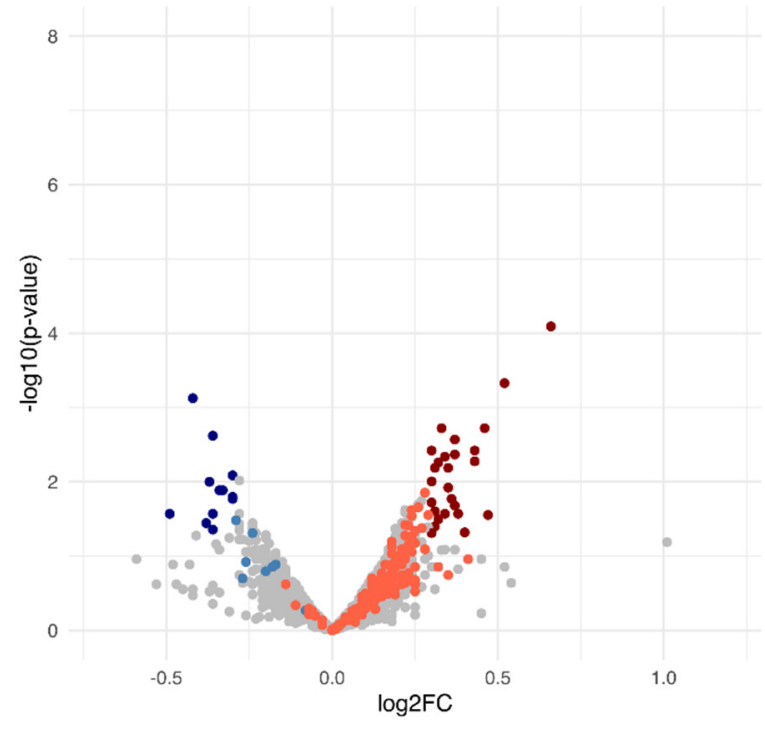

C G9a-Glp cDKO

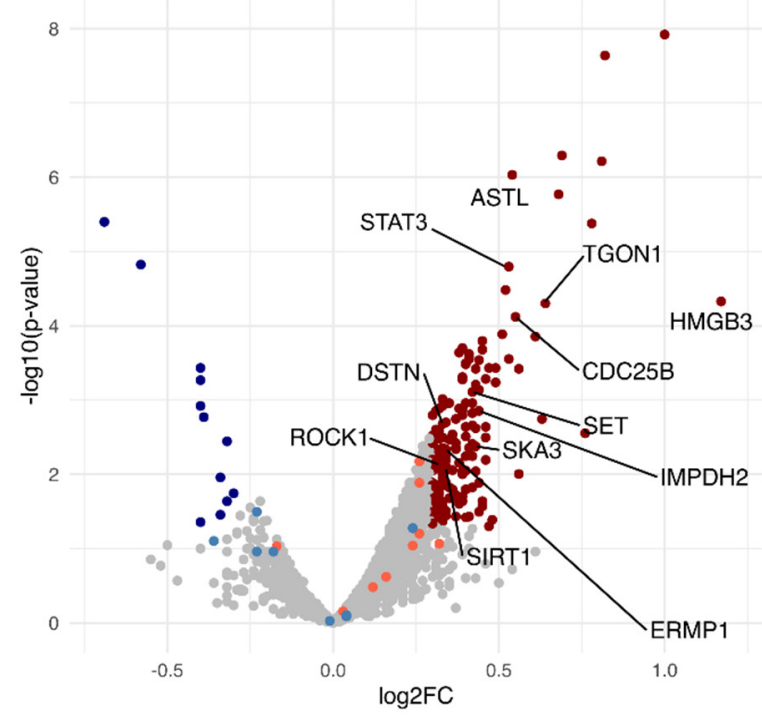


bioRxiv preprint doi: https://doi org/10.1101/2021.12.20 473460; this version posted December 21, 2021. The copyright holder for this preprint (which was not certified by peer review) is the author/funder, who has granted bioRxiv a license to display the preprint in perpetuity. It is made available under aCC-BY 4.0 International license.

\section{Figure 4}

A
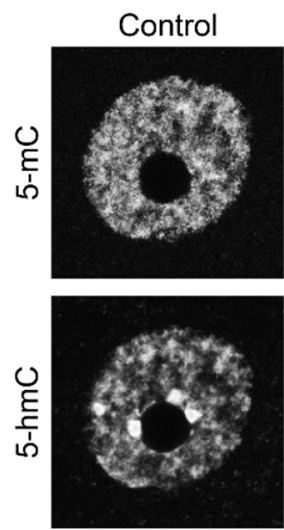

C

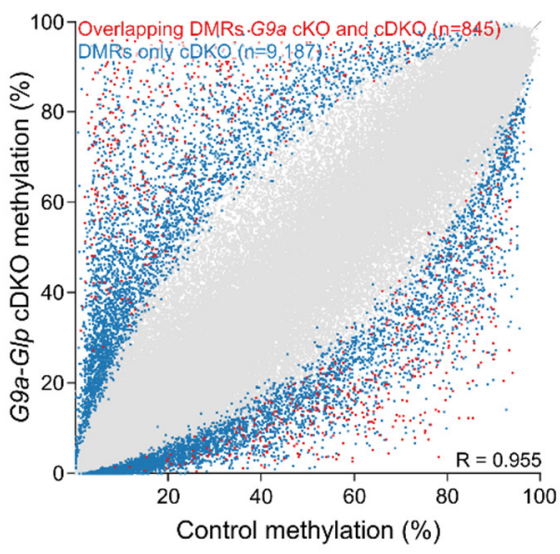

$\mathbf{F}$

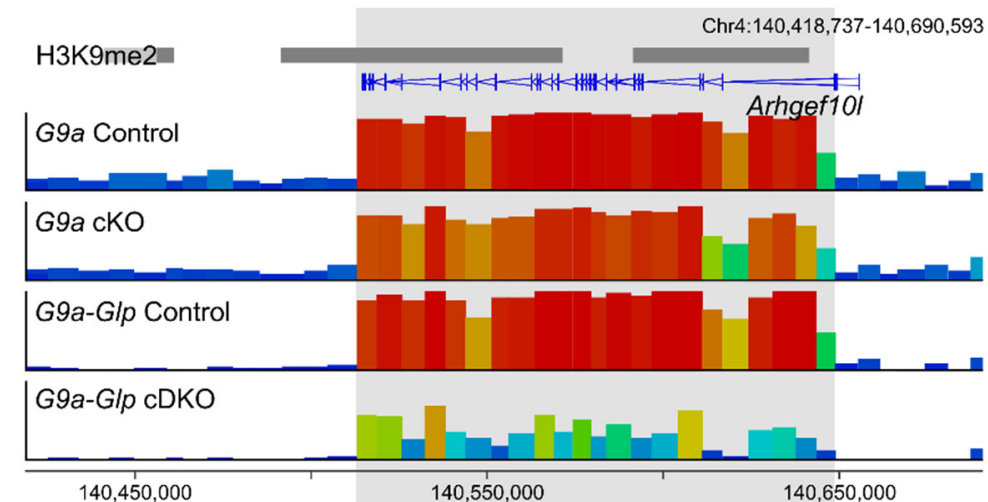

$140,450,000$
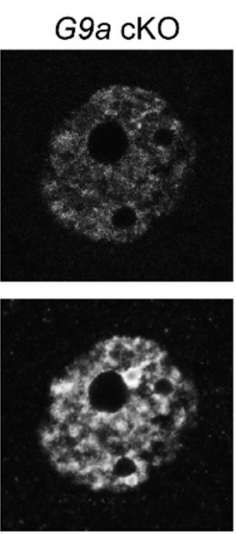

D

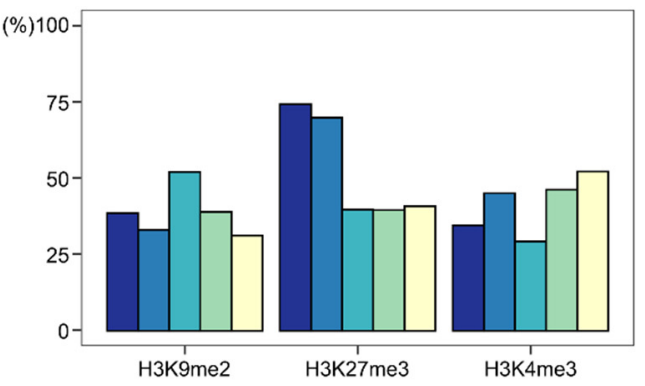

G9a cKO hyper $\square$ G9a-Glp cDKO hyper $\square$ G9a cKO hypo $\square$ G9a-Glp cDKO hypo $\square$ Random probes

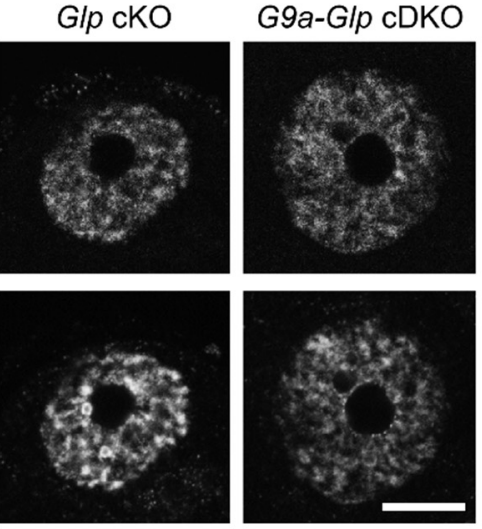

B

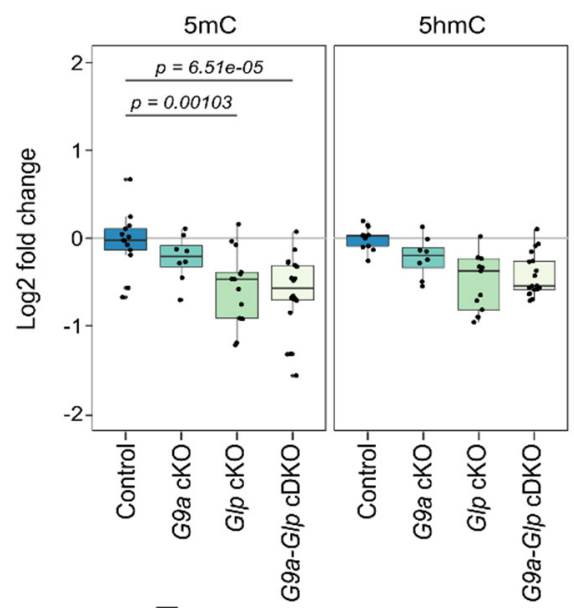

E

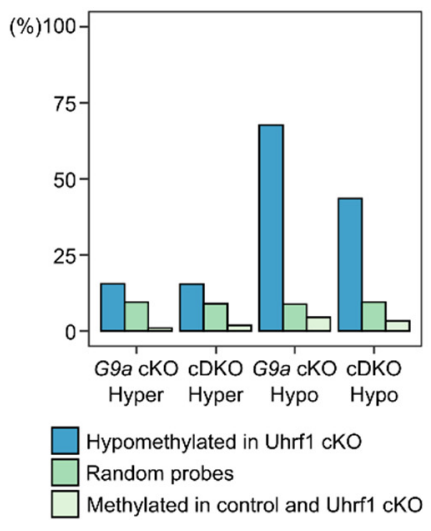

G

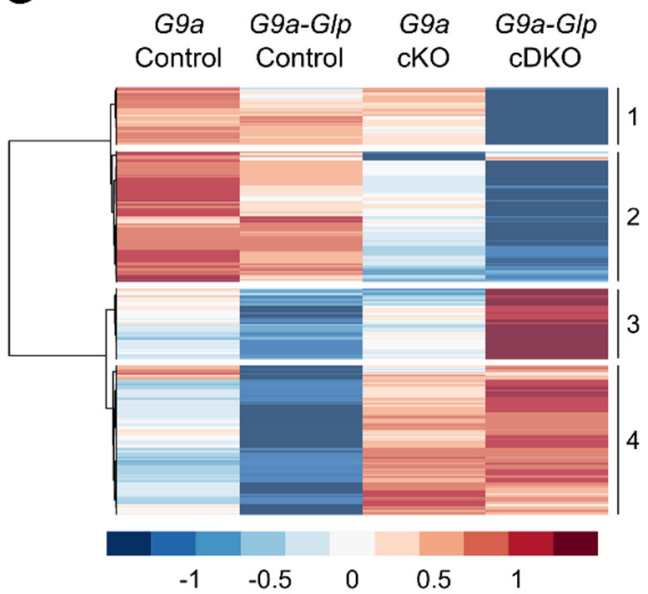


bioRxiv preprint doi: https://doi org/10.1101/2021.12 20 473460; this version posted December 21, 2021. The copyright holder for this preprint (which was not certified by peer review) is the author/funder, who has granted bioRxiv a license to display the preprint in perpetuity. It is made available under aCC-BY 4.0 International license.

\section{Figure 5}

A

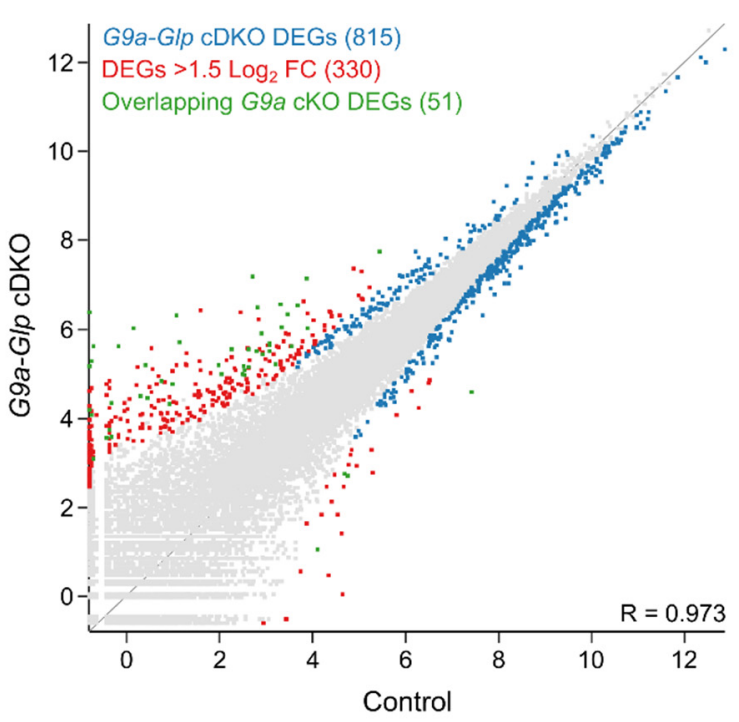

C

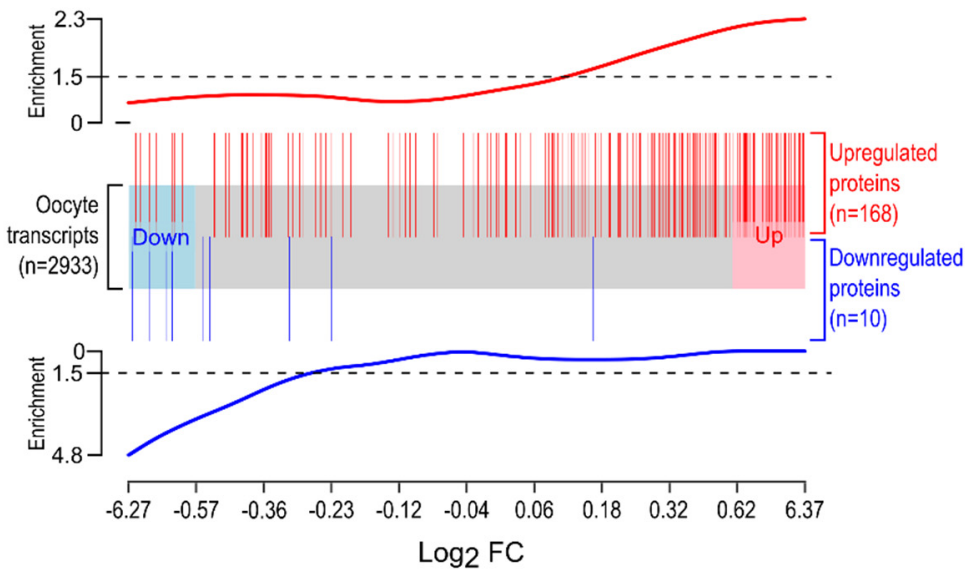

B

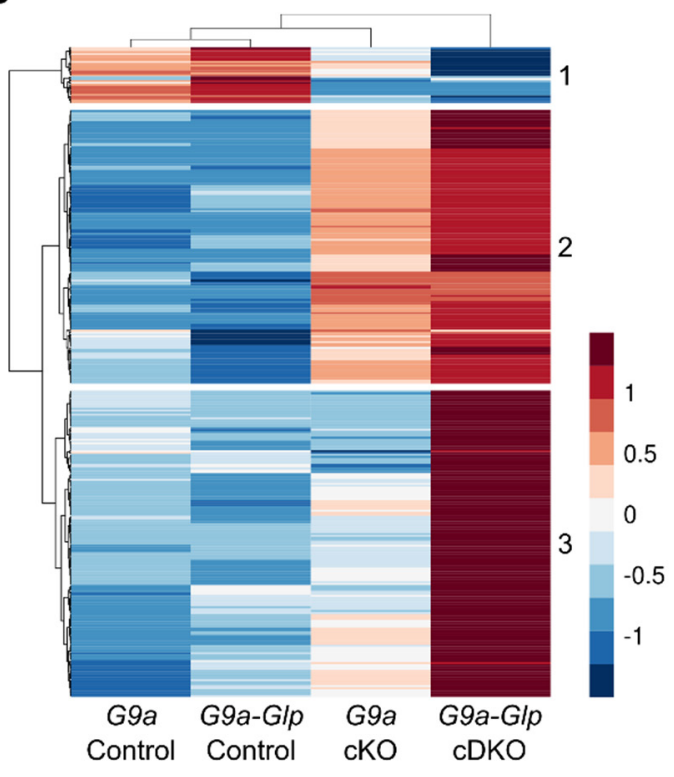

D

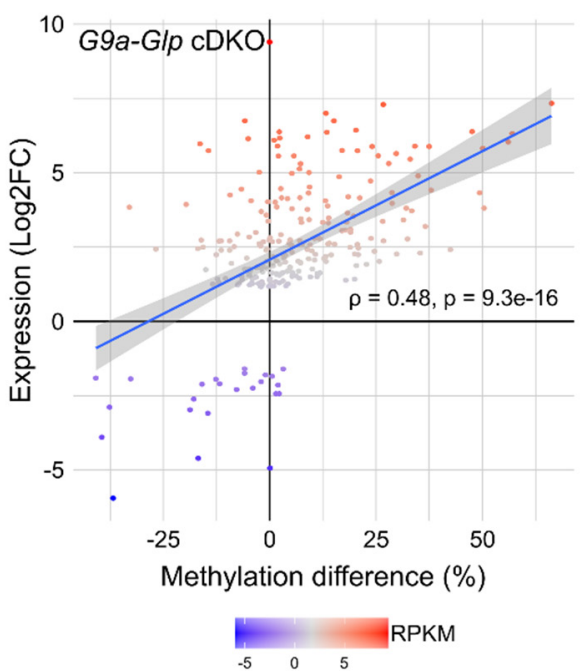


Figure 6

\section{Distinct roles for G9A and GLP in the oocyte}

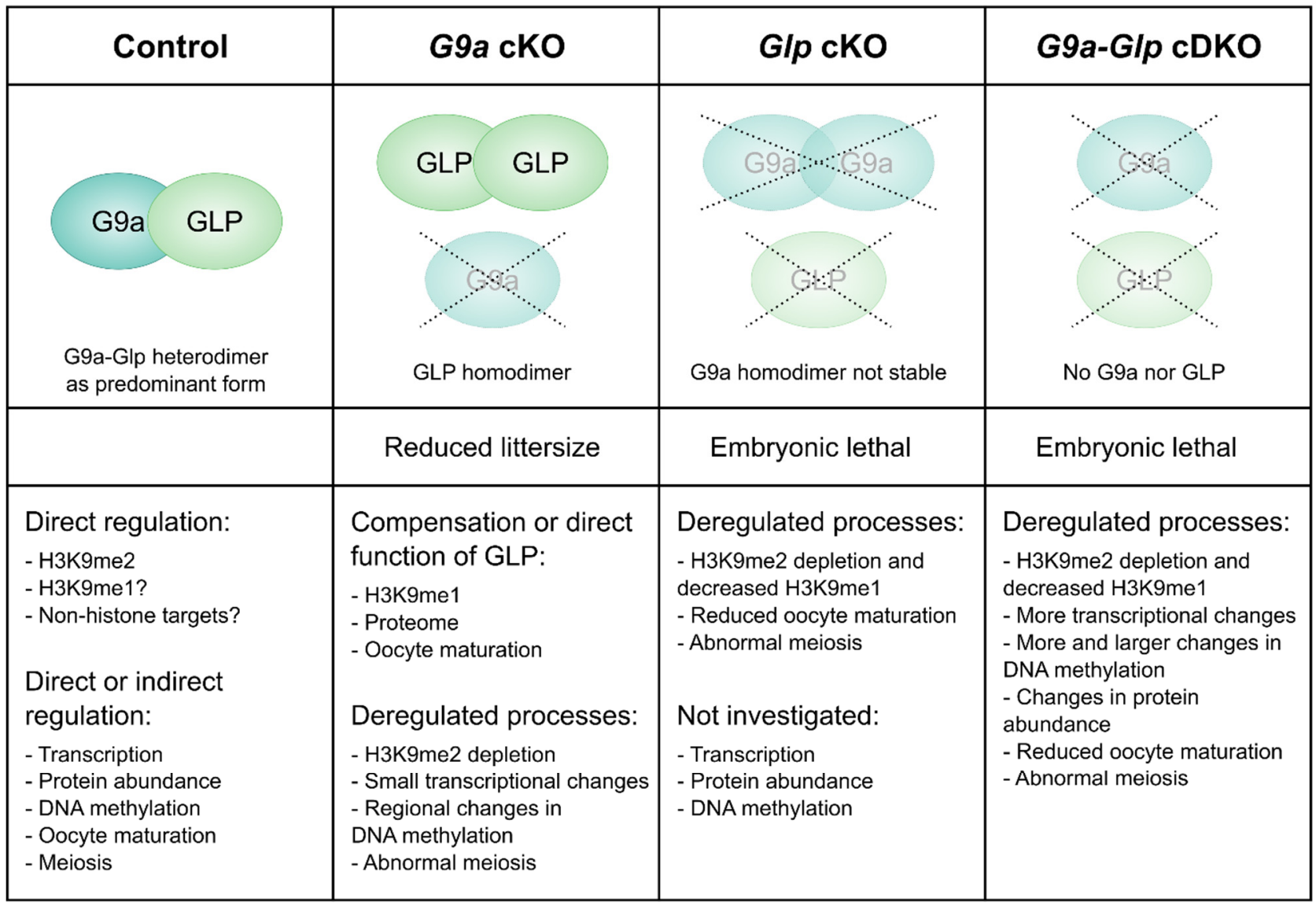

B

H3K9 methylation
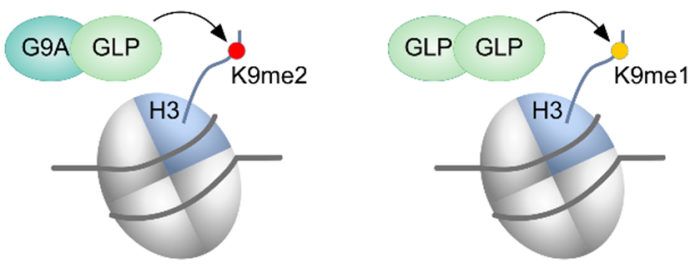

C

\section{UHRF1-dependent hypomethylation}
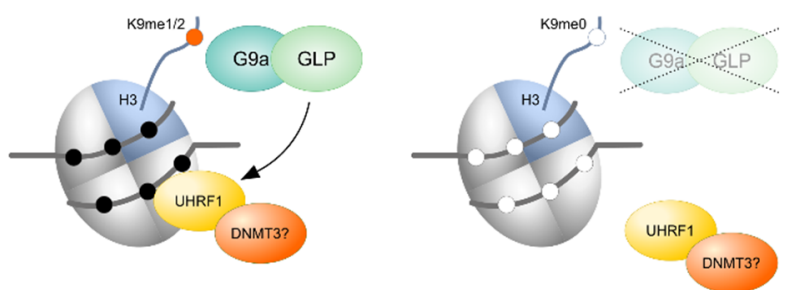

D

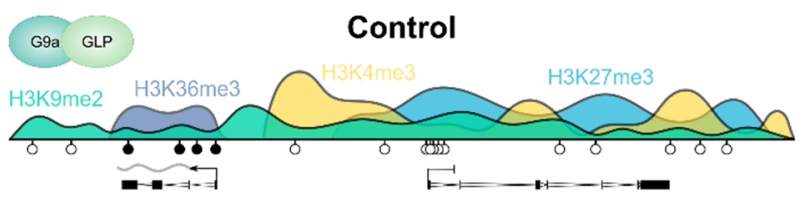

G9a cKO

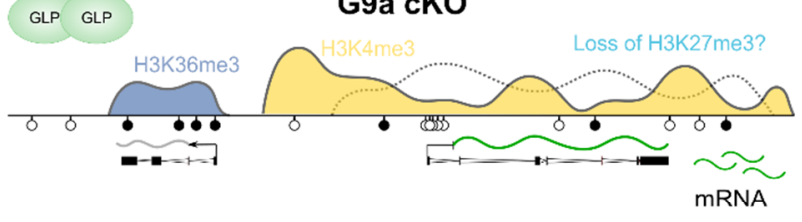

G9a-GIp cDKO

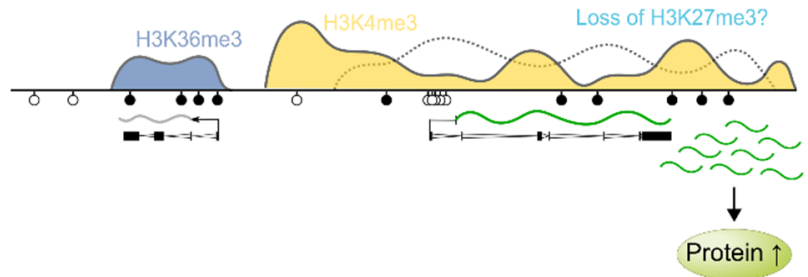




\section{Supplemental Figures}

A
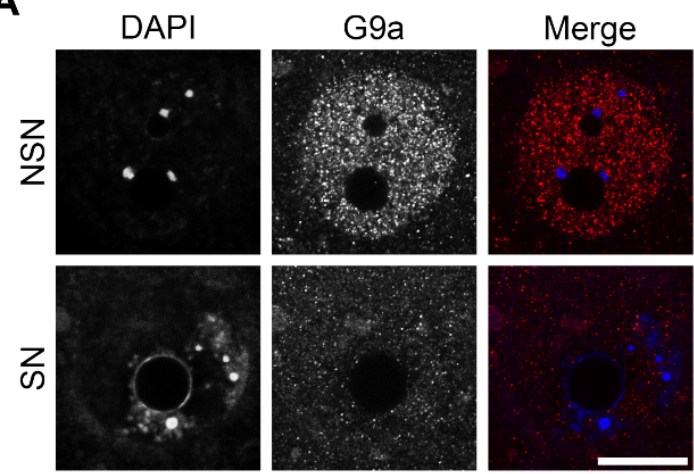

C
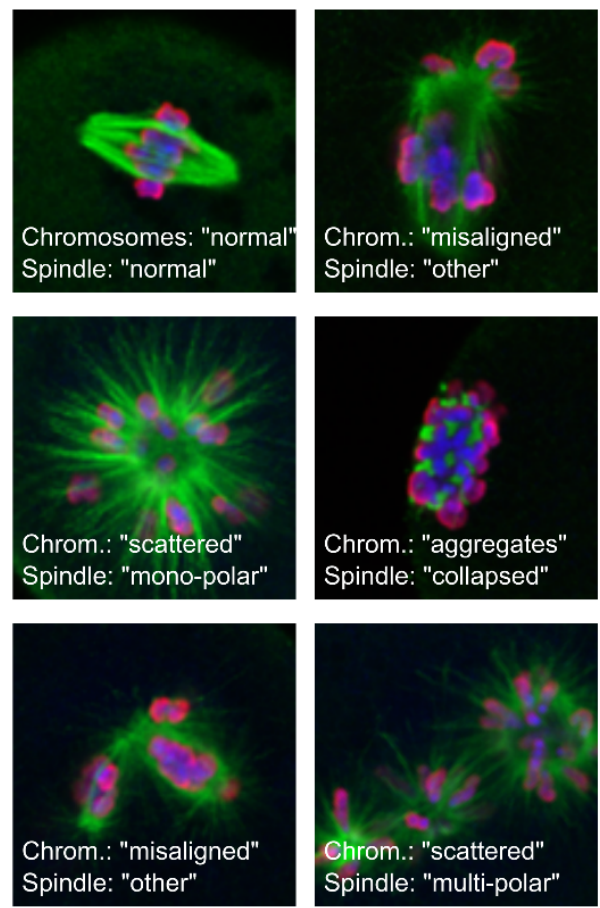

B
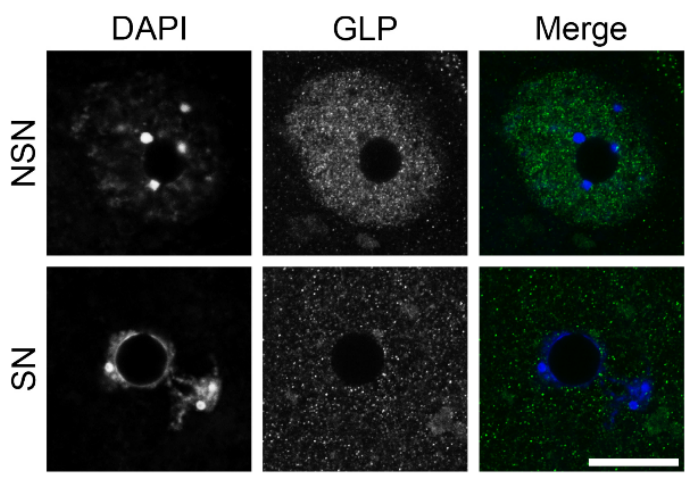

D
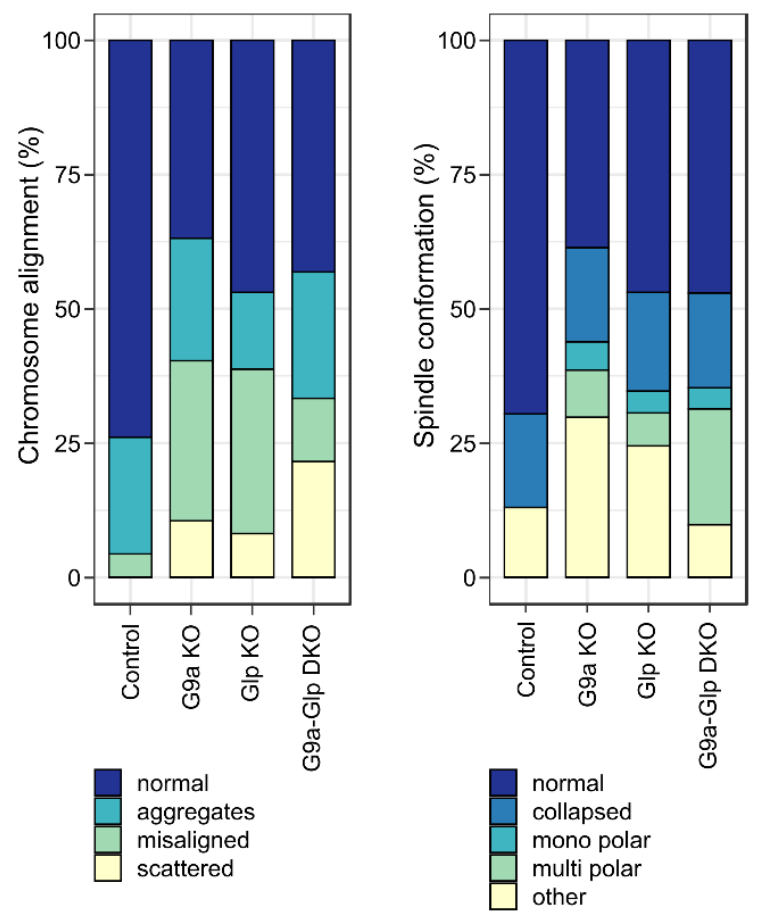

Figure S1: G9A and GLP expression in the oocyte and their effects on meiosis

A, B) Representative images showing DNA stained with DAPI, G9A expression (A) and GLP expression (B) in NSN and SN control oocytes. The scale bar represents $20 \mu \mathrm{m}$. C) Example images showing meiotic abnormalities observed in MII oocytes. The spindle is stained with an anti- $\alpha$-tubulin antibody (green) and the chromatin with DAPI (blue) and anti-pan-histone (red). D) Stacked bar charts showing the percentage of types of meiotic abnormalities (chromosome alignment and spindle conformation) observed in oocytes. 
A

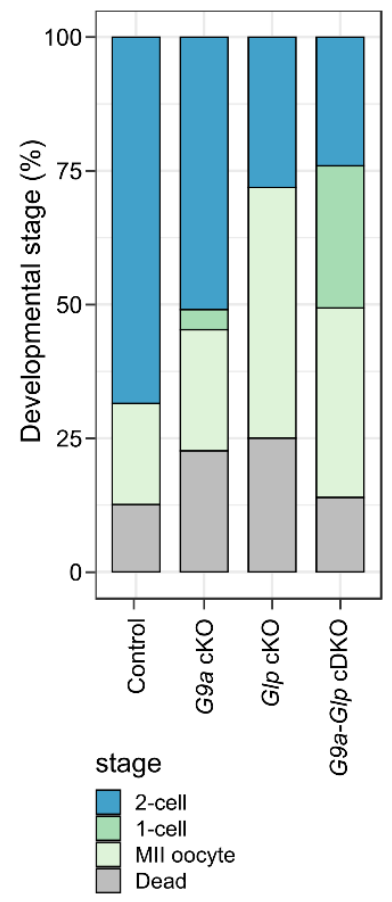

B
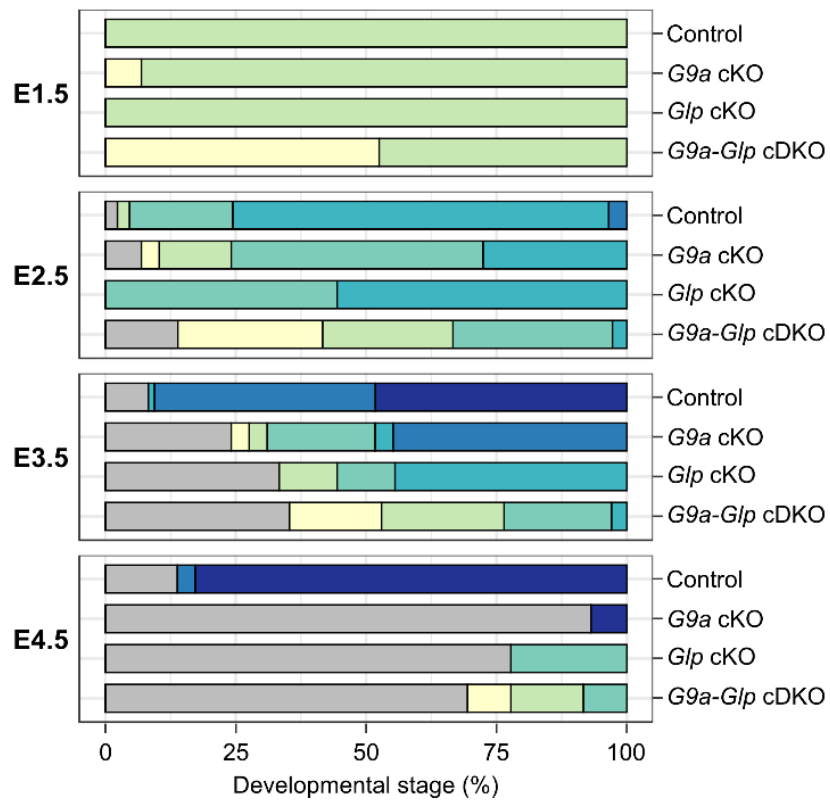

$\square$ 1-cell

8-cell

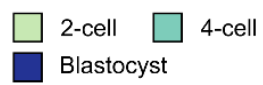

C

\section{- Control}

- G9a cKO

Glp cKO

G9a-Glp cDKO

\section{Control}

G9a cKO

GIp cKO

G9a-GIp cDKO

Control

G9a cKO

Glp cKO

G9a-Glp cDKO
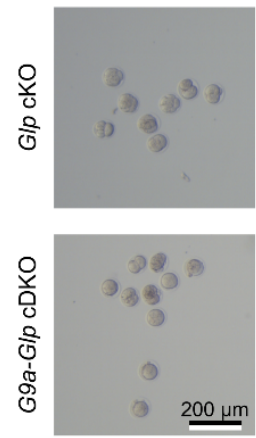

D

\begin{tabular}{lcccc}
\hline Genotype & $\begin{array}{l}\text { Normal } \\
\text { embryos }\end{array}$ & $\begin{array}{l}\text { Abnormal } \\
\text { embryos }\end{array}$ & $\begin{array}{l}\text { Resorption } \\
\text { sides }\end{array}$ & $\begin{array}{l}\text { Total } \\
\text { embryos }\end{array}$ \\
\hline Control & 7 & 1 & 0 & 8 \\
\hline G9a cKO & 7 & 2 & 0 & 9 \\
\hline G9a cKO & 4 & 4 & 0 & 8 \\
\hline Glp cKO & 0 & 3 & 0 & 3 \\
G9a-GIp cDKO & 0 & 4 & 0 & 4 \\
\hline G9a-GIp cDKO & 0 & 2 & 0 & 2 \\
\hline
\end{tabular}

E

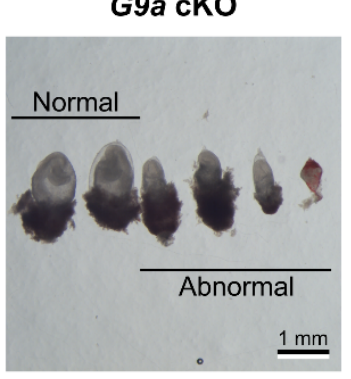

G9a-GIp cDKO

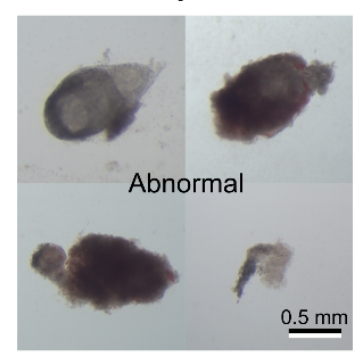

$\mathbf{F}$

\begin{tabular}{lcccc}
\hline Genotype & $\begin{array}{l}\text { Normal } \\
\text { embryos }\end{array}$ & $\begin{array}{l}\text { Abnormal } \\
\text { embryos }\end{array}$ & $\begin{array}{l}\text { Resorption } \\
\text { sides }\end{array}$ & $\begin{array}{l}\text { Total } \\
\text { embryos }\end{array}$ \\
\hline Control & 5 & 1 & 2 & $\mathbf{8}$ \\
\hline Control & 8 & 0 & 0 & $\mathbf{8}$ \\
\hline Control & 5 & 1 & 2 & $\mathbf{8}$ \\
\hline G9a cKO & 5 & 1 & 3 & 9 \\
\hline G9a cKO & 4 & 2 & 2 & $\mathbf{8}$ \\
\hline G9a cKO & 4 & 1 & 2 & $\mathbf{7}$ \\
\hline G9a-GIp cDKO & 0 & 0 & 1 & $\mathbf{1}$ \\
\hline G9a-GIp cDKO & 0 & 0 & 3 & 3 \\
\hline G9a-GIp cDKO & 1 & 0 & 1 & $\mathbf{2}$ \\
\hline
\end{tabular}

G

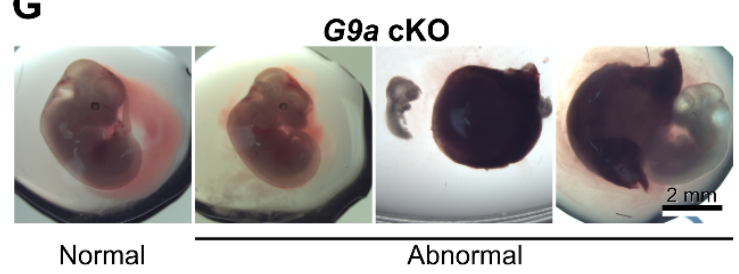

Figure S2: In vitro preimplantation and in vivo postimplantation development of embryos lacking maternal G9A and/or GLP.

A) Stacked barchart showing developmental stage of embryos collected at E1.5 after superovulation and natural mating with C57BI6/Babr WT males. One-way ANOVA with Tukey's posthoc test: 2-cell stage Control vs G9a-GIp cDKO P $=0.0068^{* *}$. B) Stacked barcharts showing developmental stage of embryos cultured in vitro after natural mating on E1.5, E2.5, E3.5 and E4.5. One-way ANOVA with Tukey's posthoc test: morula stage Control vs G9a cKO $P=0.0006^{* * *}$. C) Images showing examples of embryos cultured in vitro on E3.5. D, E) Table showing embryo development at E8.5 (D) and E12.5 (E) after natural mating of conditional KO females with WT males. Each line represents one female mouse. Indicated are the number of normal embryos, abnormal embryos and resorption sides for each female. 
A

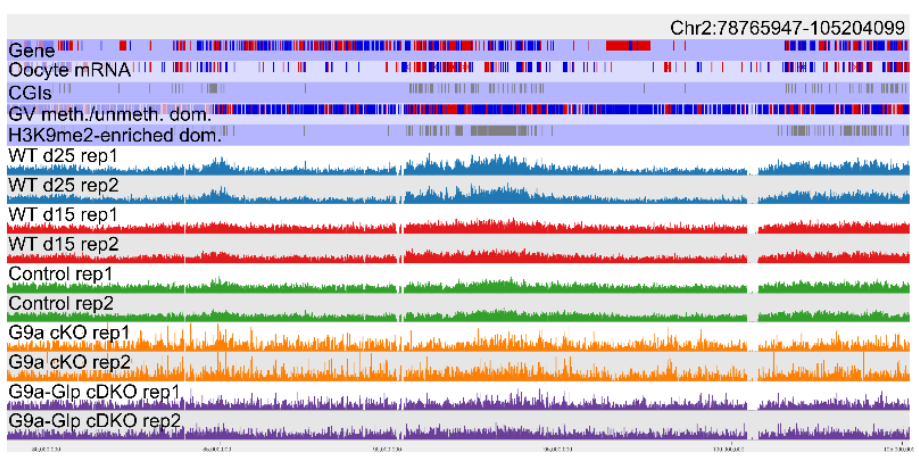

B

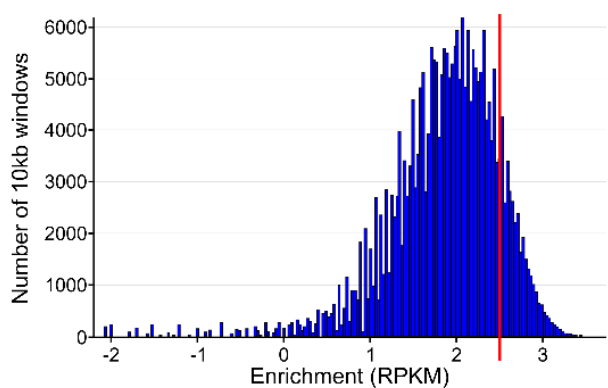

C

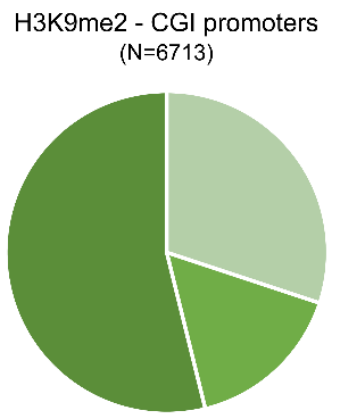

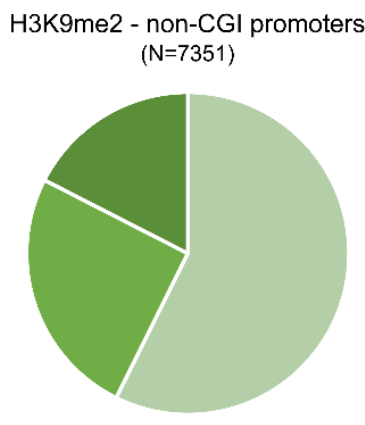

FPKM $<0.1$
Random - CGI promoters $(\mathrm{N}=2163)$

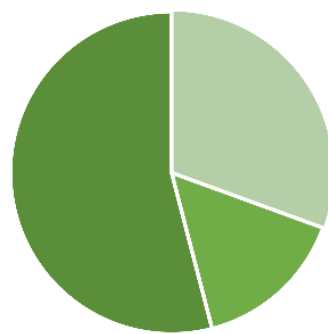

FPKM > 1
Random - non-CGI promoters $(\mathrm{N}=11904)$

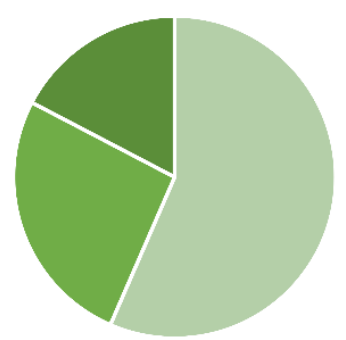

FPKM 0.1-1

Figure S3: ChIP-seq analysis showing H3K9me2 distribution in control, G9a cKO and G9a-GIp cDKO oocytes.

A) Screenshot showing H3K9me2 enrichment and reproducibility of replicates. B) Histogram showing number of $10 \mathrm{~kb}$ windows with a certain enrichment level in d25 WT oocytes. The red line indicates the threshold set to define H3K9me2 enrichment (RPKM > 2.5). C) Pie charts showing overlap of H3K9me2 enriched and random probes with untranscribed (FPKM $<0.1$ ), lowly transcribed (FPKM 0.1-1) and highly transcribed genes (FPKM $>1$ ). 
A

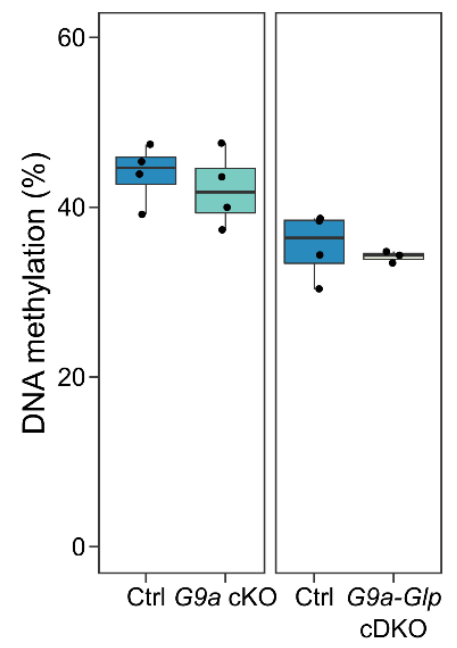

D

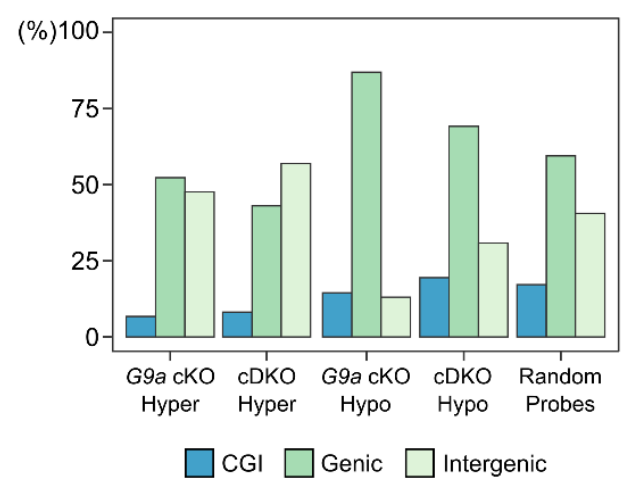

$\mathbf{F}$

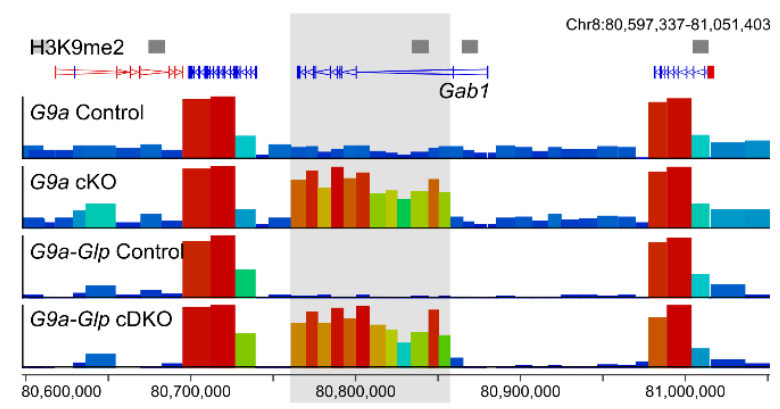

E

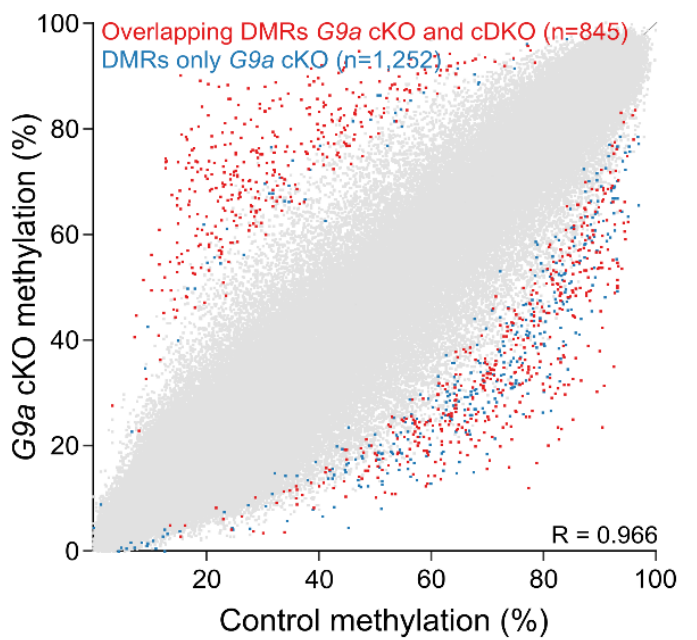

Hypermethylated DMRs

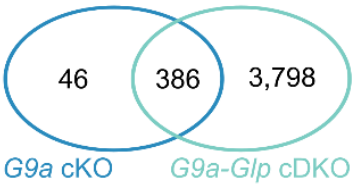

Hypomethylated DMRs

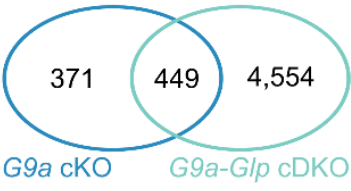

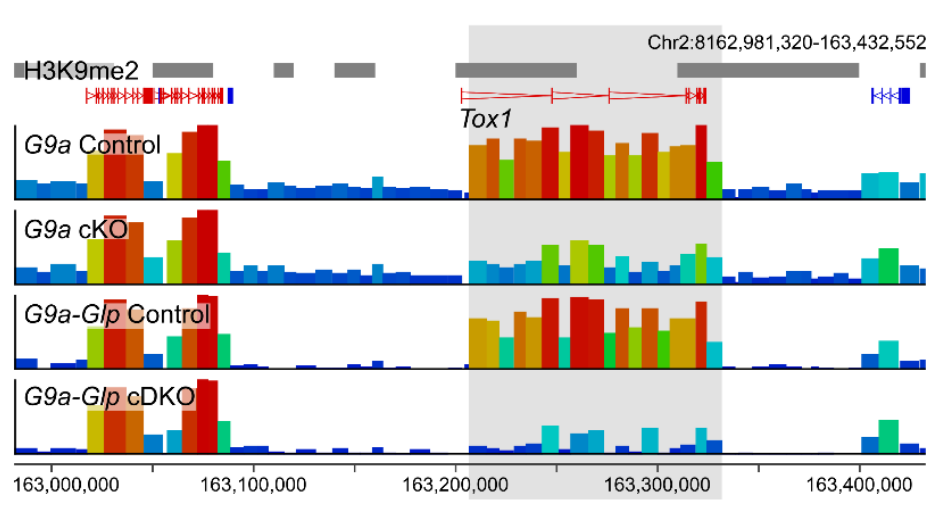

G

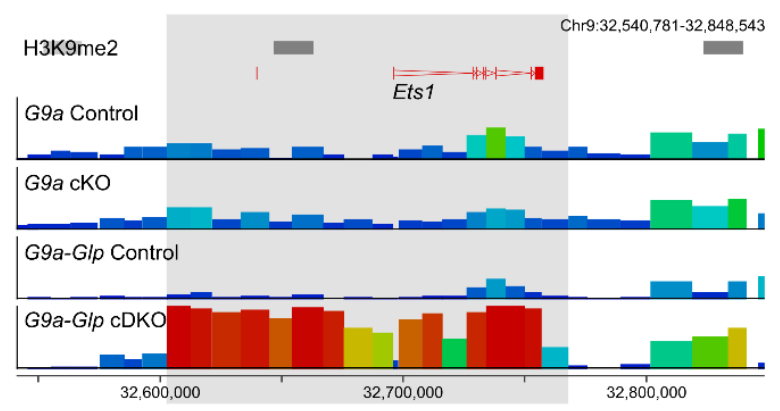

Figure S4: DNA methylation analysis of G9a cKO and G9a-GIp cDKO oocytes with corresponding littermate controls.

A) Box-whisker plots showing global DNA methylation levels using average methylation levels of $100 \mathrm{CpG}$ windows. Ctrl vs G9a cKO $P=0.5378$; Ctrl vs G9a-Glp cDKO $P=0.6077$ B) Scatterplot showing methylation levels of control vs G9a cKO oocytes. Unique G9a cKO DMRs are highlighted in blue and DMRs shared with G9a-Glp cDKO oocytes in red. C) Venn diagrams, showing overlap of hypermethylated and hypomethylated DMRs between G9a cKO and G9a-Glp cDKO oocytes. D) Barchart showing percentage of DMRs and random probes overlapping CGIs, genic and intergenic regions. Qi-square comparing genic and intergenic DMRs: G9a cKO adj. $P=0.026^{*}$; all others $P<0.0001^{* * *}$. E-G) Genome screenshots showing examples differentially methylated domains. Shown are a region that is hypomethylated in both G9a cKO and G9aGlp cDKO oocytes (E), a region that is hypermethylated in both G9a cKO and G9a-G/p cDKO oocytes (F) and a region that is uniquely hypermethylated in G9a-Glp cDKO oocytes (G). 
A

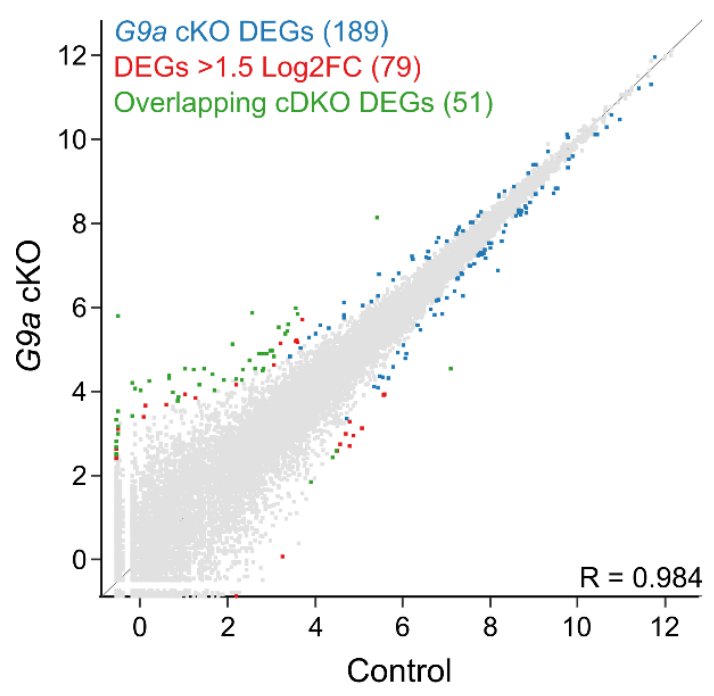

B

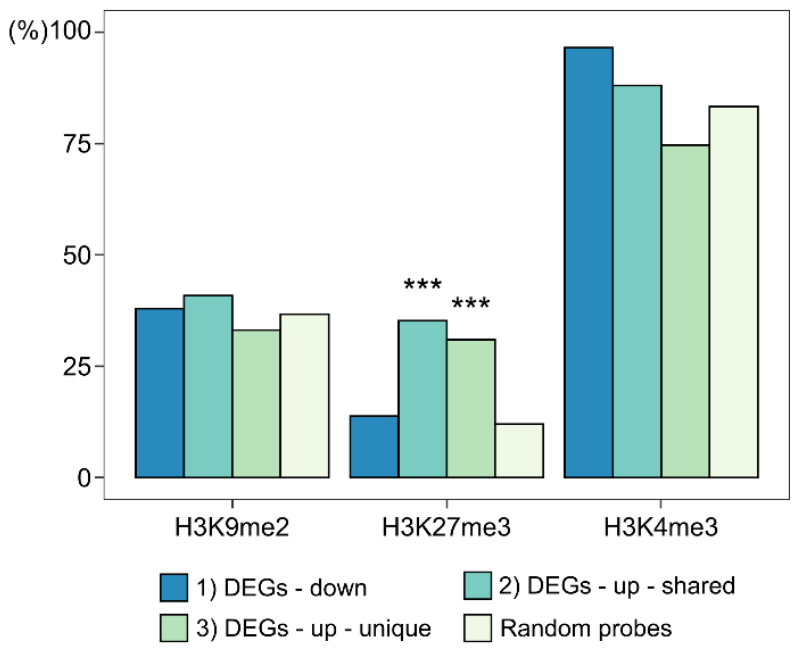

E

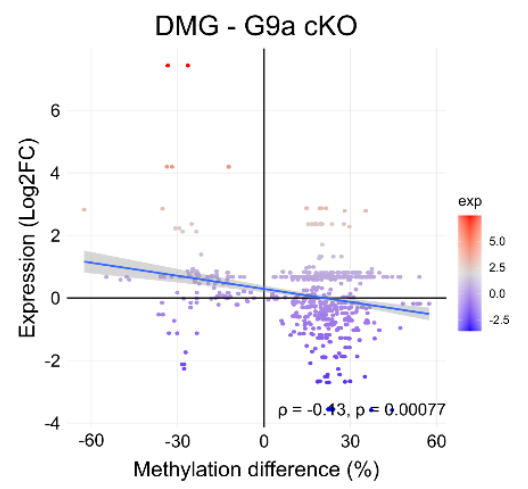

Figure S5: RNA-seq analysis showing transcriptome changes in G9a cKO and G9a cDKO oocytes and their link with DNA methylation.

A) Scatterplot showing expression levels of control vs. G9a cKO oocytes. Unique G9a cKO DEGs are highlighted in blue, DEGs with Log2FC $>1.5$ in red and DEGs shared with G9a-Glp cDKO oocytes in green. B) Barchart showing proportion of DEGs overlapping with histone modifications. DEGs are split according to the clustering analysis of Figure 5B. Qi-square analysis comparing DEGs to random probes: $P<0.0001^{* * *}$. C) Plot showing link between methylation and expression changes of differentially methylated genes in G9a-Glp cDKO oocytes. D, E) Plot showing link between methylation and expression changes of differentially expressed genes (C) and differentially methylated genes (D) in G9a cKO oocytes. C-E) Expression levels are indicated by the colour scale. Spearman correlation indicated in plot. 